Portland State University

PDXScholar

$12-2-2016$

\title{
Development of 3D Accelerometer Testing System
}

Mohammed Abbas Soudai Alsaedi

Portland State University

Follow this and additional works at: https://pdxscholar.library.pdx.edu/open_access_etds

Part of the Mechanical Engineering Commons

Let us know how access to this document benefits you.

\section{Recommended Citation}

Alsaedi, Mohammed Abbas Soudai, "Development of 3D Accelerometer Testing System" (2016).

Dissertations and Theses. Paper 3371.

https://doi.org/10.15760/etd.5262

This Thesis is brought to you for free and open access. It has been accepted for inclusion in Dissertations and Theses by an authorized administrator of PDXScholar. Please contact us if we can make this document more accessible: pdxscholar@pdx.edu. 
Development of 3D Accelerometer Testing System

by

Mohammed Abbas Soudai Alsaedi

A thesis submitted in partial fulfillment of the requirements for the degree of

\author{
Master of Science \\ in \\ Mechanical Engineering
}

\begin{abstract}
Thesis Committee:
Sung Yi, Chair

Chien Wern

David Turcic
\end{abstract}

Portland State University

2016 


\begin{abstract}
Accelerometers are used in numerous industries including aircrafts and missile navigations systems, rotary machines, and electronic devices such as tablets, cell phones, and cameras. Accelerometers of different purposes and specifications are being produced in large quantities. The large demand for accelerometers forces the need for accelerometer testing methodology that is more convenient and accurate. Inertial Micro-Electromechanical Systems Accelerometer (MEMS) require a series of tests that include physical stimuli. One of the key challenges is the cost associated with testing. Therefore, the accurate prediction of the sensor functions not only reduces the testing time but also more importantly contributes to reducing testing cost.

Shaker accelerometer calibrators are widely used to test accelerometers. Shakers use sinusoidal acceleration input, and their testing acceleration range could be as small as $0.1 \mathrm{~g}^{1}$ and as high as $20 \mathrm{~g}$. These devices test accelerometers in one axis at a time. In this study, the 3-D MEMS accelerometer testing method and system have been developed to reduce the testing time cycle significantly by testing 3D axes simultaneously. The theoretical study shows that an acceleration sensor is rotating about a fixed axis experiences tangential and normal accelerations.

The objective of this project is to develop a testing methodology and equipment to test accelerometers along all three axes simultaneously over a range of $\pm 50 \mathrm{~g}$ while reducing testing time. These accelerations are related to the distance from the center of rotation and the angular velocity. The angular velocity can be controlled by the amount of the voltage that is given to the DC motor from the power supply which is adjusted by a speed controller. By varying either the angular velocity with the speed

\footnotetext{
${ }^{1} \mathrm{~g}($ Gravity $)=9.8 \mathrm{~m} / \mathrm{s}^{2}$
} 
controller or varying the distance from the axis of rotation, it is possible to expose an accelerometer to the desired acceleration value.

The equipment uses a rotating turntable where the center of the table marks the axis of the rotation. The accelerometer being tested was mounted at a known distance from the center of the table. The resulted normal and tangential accelerations were calculated from the angular velocity of the turntable and the position of accelerometer from the axis of rotation. A high precision encoder was used to determine the angular velocity of the turntable. The range of the angular velocity that can be measured accurately by the encoder affects the range of acceleration amplitude the system can be used to test. The encoder used can sense an angular velocity as low as $0.0144^{\circ} / \mathrm{s}$. This corresponds to the normal acceleration value of $2.32 \times 10^{-9} \mathrm{~g}$.

The accelerometer mounted on a turntable in a way that all three of its axes are exposed to the input acceleration to test an accelerometer in all three axes at the same time. A program written in LabVIEW operated the system and collected the test data. This program was able to collect acceleration readings along all three axes of the accelerometer with position and velocity information of the input motion. The collected data set were processed by MATLAB. This testing methodology provides a way to use multiple input accelerations unlike traditional accelerometer testing method. The test methodology developed in this study provides a way to use a constant acceleration input over a wide range $( \pm 50 \mathrm{~g})$. Its lowest acceleration range is $2.32 \times 10^{-9} \mathrm{~g}$. This reduces the time it takes to align the accelerometer in different axes and eliminates errors that may result during a manual repositioning of the accelerometer to align it in a different axis. Error models for MEMS accelerometer sensors have used Kalman filters to perform complete accelerometer sensor test. The accelerometer error model must be constructed, and the coefficients in the error equations must be determined. Therefore, Kalman filter in the analysis of visual 
motion has been documented frequently. The filter is constructed as a mean squared error minimizer. The purpose of filtering is to extract the required information from a signal, ignoring everything else. 


\section{Acknowledgements}

First of all, many thanks to Allah (God) for giving me the faith and strength to finish this scientific work. I would like to express my sincere gratitude to Professor Sung Yi, the supervisor and the chair of the thesis committee, for his generous help, support, and countless time of reviewing, reading, and advising. I also like to extend my appreciation to Professors Chien Wern and David Turcic for serving as committee members and reviewing this thesis. This piece of science became more informative and practically useful with their comments and recommendations. My sincere gratitude to

my parents, sisters, brothers, and wife for the emotional and financial support during my academic journey. I also like to thank Craig Boxrucker, the student who started with this project. Special thanks to all graduate students at Portland State University (PSU) who helped me with experimental work, especially Salih Mahmood, Hayder Al-Khafaji, Ali Zerkane, Aqeel Al-Bahadily. My gratitude to the Higher Committee for Education Development in Iraq (HCED). 


\section{Table of Contents}

Abstract

Acknowledgements $\quad$ iv

List of Tables $\quad$ viii

List of Figures $\quad$ ix

Nomenclature $\quad 1$

$\begin{array}{lll}\text { Chapter } 1 & \text { Indroduction } & 1\end{array}$

1.1 Introduction to Accelerometers . . . . . . . . . . . . . . . . . . 1

1.1.1 Piezoelectric Crystal . . . . . . . . . . . . . . 1

1.1.2 Micro-Electromechanical Systems Accelerometer (MEMS) . . 3

1.2 Scope ............................ 6

1.3 Outline of Study . . . . . . . . . . . . . . . . . . . . 7

Chapter 2 Testing Methodology and Error Analysis of MEMS Ac$\begin{array}{lr}\text { celerometer } & 8\end{array}$

2.1 Generation of Acceleration Input . . . . . . . . . . . . . . . . 10

2.1.1 Single Axis Test Mode . . . . . . . . . . . . . . . . . 10

2.1.2 Tri-Axis Test Mode . . . . . . . . . . . . . . . . . 24 
$\begin{array}{lll}\text { Chapter } 3 & \text { Test Equipment Design and Setup } & 39\end{array}$

3.1 Design Description . . . . . . . . . . . . . . . 39

3.1.1 Turntable ...................... . . 40

3.1.2 Mounting the Sensor . . . . . . . . . . . . . . . . . 41

3.1.3 Wiring the Turntable ................ . 41

3.1.4 Motor Selection .................. 42

3.1.5 Accelerometer Sensor . . . . . . . . . . . . . . 43

3.2 Velocity Measurement Device Selection . . . . . . . . . . . . . . 44

3.3 Kinematic Model . . . . . . . . . . . . . . . . . . . . . . . . . 48

3.4 The Testing Methodology of Simulation . . . . . . . . . . . . . 52

3.5 Verification Study _. . . . . . . . . . . . . . . . 53

3.5.1 Kalman Filter Parameters . . . . . . . . . . . . . 53

3.5.2 Static Testing-Balance ............. 53

Chapter 4 Results of Error Analysis of MEMS Accelerometer Sen$\begin{array}{lc}\text { sor and Testing } & 56\end{array}$

4.1 Three-dimensional Testing System. . . . . . . . . . . . . . . 58

4.2 Data Processing . . . . . . . . . . . . . . . 60

4.3 3-D Testing Results \& Discussion . . . . . . . . . . . . . . . 61

4.3.1 Measured and Output ................... 61

4.3.2 Estimation of Errors . . . . . . . . . . . 66

4.4 Effect the Misalignment of the Turntable on the Results . . . . . . . 71

Chapter 5 Conclusion, Recommendation and Future Works. $\quad 72$

5.1 Summary and Conclusion . . . . . . . . . . . . . . 72

5.2 Future work and recommendation . . . . . . . . . . . . 73 
References

Appendix A Glossary of MEMS Accelerometer Testing Machine Terminology

Appendix B Labview ${ }^{M T}$ Program 


\section{List of Tables}

Table 3.1 Properties of the DC motor . . . . . . . . . . . . . . . . 42

Table 3.2 Analog inertial sensor (ADIS16400) specification . . . . . . . . 43

Table 4.1 Acceleration testing machine properties . . . . . . . . . . . 59

Table 4.2 Summary of 3-D testing results . . . . . . . . . . . . . . . 71 


\section{List of Figures}

Figure 1.1 Spring and Damper System in an Accelerometer [1] . . . . . 1

Figure 1.2 Electronic Circuit $[2] \ldots \ldots$. . . . . . . . . . . . . 3

Figure 1.3 3D MEMS Accelerometer Packaging [3] . . . . . . . . . . . 5

Figure 2.1 Scheme of Testing Accelerometer . . . . . . . . . . . . . . . . 9

Figure 2.2 Single Axis Test about $x$ Axis . . . . . . . . . . . . . . . . 11

Figure 2.3 Single Axis Test about $y$ Axis . . . . . . . . . . . . . . . . . . 12

Figure 2.4 Single Axis Test about $z$ Axis . . . . . . . . . . . . . . . . . . 13

Figure 2.5 Output vs. Input Acceleration Along $i$-Axis . . . . . . . . . . 17

Figure 2.6 Output Acceleration Along $j$-Axis vs. Input Acceleration Along

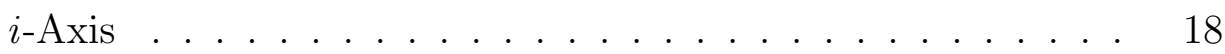

Figure 2.7 Deviations of the Data From the Estimated Regression Model [4] 19

Figure 2.8 Position of the Sensor for Tri Axes Test . . . . . . . . . . . . . 24

Figure 2.9 Scheme of Testing 3D-Axes Accelerometer . . . . . . . . . . . 26

Figure 2.10 Transform Global Coordinates to the Local Coordinates . . . . 27

Figure 2.11 A Complete Picture of the Operation of Kalman Filter [5] . . 36

Figure 3.1 General Idea of the Test . . . . . . . . . . . . . . . . . . . . 39

Figure 3.2 Dimensions of the Turntable . . . . . . . . . . . . . . . . . . . 40

Figure 3.3 Limit Acceleration by Adjusting Radius . . . . . . . . . . . . 41

Figure 3.4 Virtual Absolute Encoder . . . . . . . . . . . . . . . 45 
Figure 3.5 Principle of Velocity Measurement Method [6] . . . . . . . . . 46

Figure 3.6 The Euler Angles $[7]$. . . . . . . . . . . . . . . . . . . . . . . . . . . . 49

Figure 3.7 Shaft Balance . . . . . . . . . . . . . . . . . . . . . . 54

Figure 3.8 Balance of the Turntable with Accelerometer Sensor . . . . . . 55

Figure 3.9 Static Balance Testing of the Turntable . . . . . . . . . . . 55

Figure 4.1 Common Errors in Accelerometer Sensor [8] . . . . . . . . . . 57

Figure 4.2 3D Testing Machine . . . . . . . . . . . . . . . . . . . . . 58

Figure 4.3 Acceleration Input and Sensor Output about $x$-Axis vs. Time 61

Figure 4.4 Acceleration Input and Sensor Output about $y$-Axis vs. Time 62

Figure 4.5 Acceleration Input and Sensor Output about $z$-Axis vs. Time 63

Figure 4.6 Measured Acceleration around $x, y$ and $z$ Axes with the Kalman

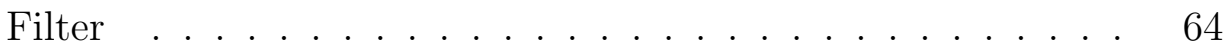

Figure 4.7 Sensor Outputs Along $x, y$ and $z$ Axes with the Kalman Filter 65

Figure 4.8 Output Acceleration around $x$-Axis with Raw Data and Kalman Filter ..................... 66

Figure 4.9 Sensor Bias Estimation Along $x, y$, and $z$ Axes . . . . . . . 67

Figure 4.10 Scale Factor Estimation Along $x, y$, and $z$ Axes $\ldots . . . . .68$

Figure 4.11 Sensor Misalignment Estimations Along x, y, and z Axes . . . 70 


\section{Chapter 1}

\section{Indroduction}

\subsection{Introduction to Accelerometers}

An accelerometer is a device that measures acceleration forces. These forces may be static, like the constant force of gravity, or they could be dynamic. The dynamic acceleration force could be caused by moving or vibrating the accelerometer. There are many types of accelerometers sensors, but the most common ones are as the followings.

\subsubsection{Piezoelectric Crystal}

This type of accelerometer is big in size [9].

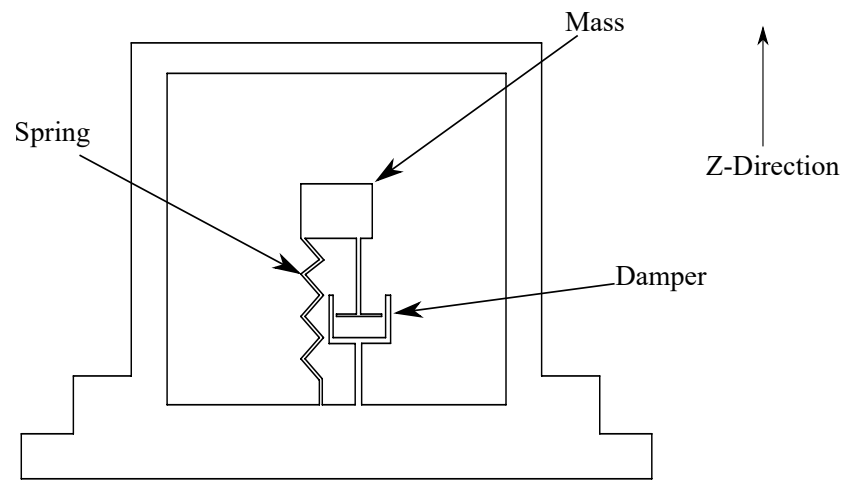

Figure 1.1: Spring and Damper System in an Accelerometer [1] 
The working principle of the accelerometer can be described by a single mass $m$ attached to a spring of stiffness $k$, which in turn is connected to a casing as illustrated in Fig. 1.1. The mass used in accelerometers is often called the seismic-mass or proofmass. The system also includes a dashpot to provide a desirable damping effect. The dashpot with a damping coefficient $c$ is usually attached to the mass in parallel with the spring. When the spring mass system is subjected to a linear acceleration, a force equal to mass times acceleration acts on the proof-mass, causing it to deflect. This deflection is sensed by a suitable means and converted into an equivalent electrical signal. Some form of damping is required. Otherwise, the system would not stabilize quickly under applied forces. To derive the equation of motion of the system, Newton's second law is used [1]. From the stationary observer's point of view, as shown in Fig. 1.1 the sum of all forces in the $\mathrm{Z}$ direction is:

$$
\begin{gathered}
\mathbf{F}_{\text {applied }}-\mathbf{F}_{\text {damping }}-\mathbf{F}_{\text {spring }}=m \ddot{\mathbf{D}} \\
m \ddot{\mathbf{D}}+\mathbf{F}_{\text {damping }}+\mathbf{F}_{\text {spring }}=\mathbf{F}_{\text {applied }} \\
m \ddot{\mathbf{D}}+c \dot{\mathbf{D}}+k \mathbf{D}=\mathbf{F}
\end{gathered}
$$

where:

$m=$ mass of the proof-mass.

$\mathbf{D}=$ relative movement of the proof-mass on frame.

$c=$ damping coefficient of damper.

$k=$ spring constant.

$\mathbf{F}=$ force applied. 


\subsubsection{Micro-Electromechanical Systems Accelerometer (MEMS)}

Recently, researchers have been working to develop a new accelerometer sensor that could increase applicability. MEMS accelerometer is considered the first micro machined accelerometer was designed in 1979 at Stanford University [9]. After 15 years, MEMS accelerometers started to be widely used in many industries like the automotive-airbag system. MEMS system consisting of a cantilever beam with a proof-mass (also known as seismic-mass), is placed in single crystal silicon using surface micromachining or bulk micromachining processes [10].

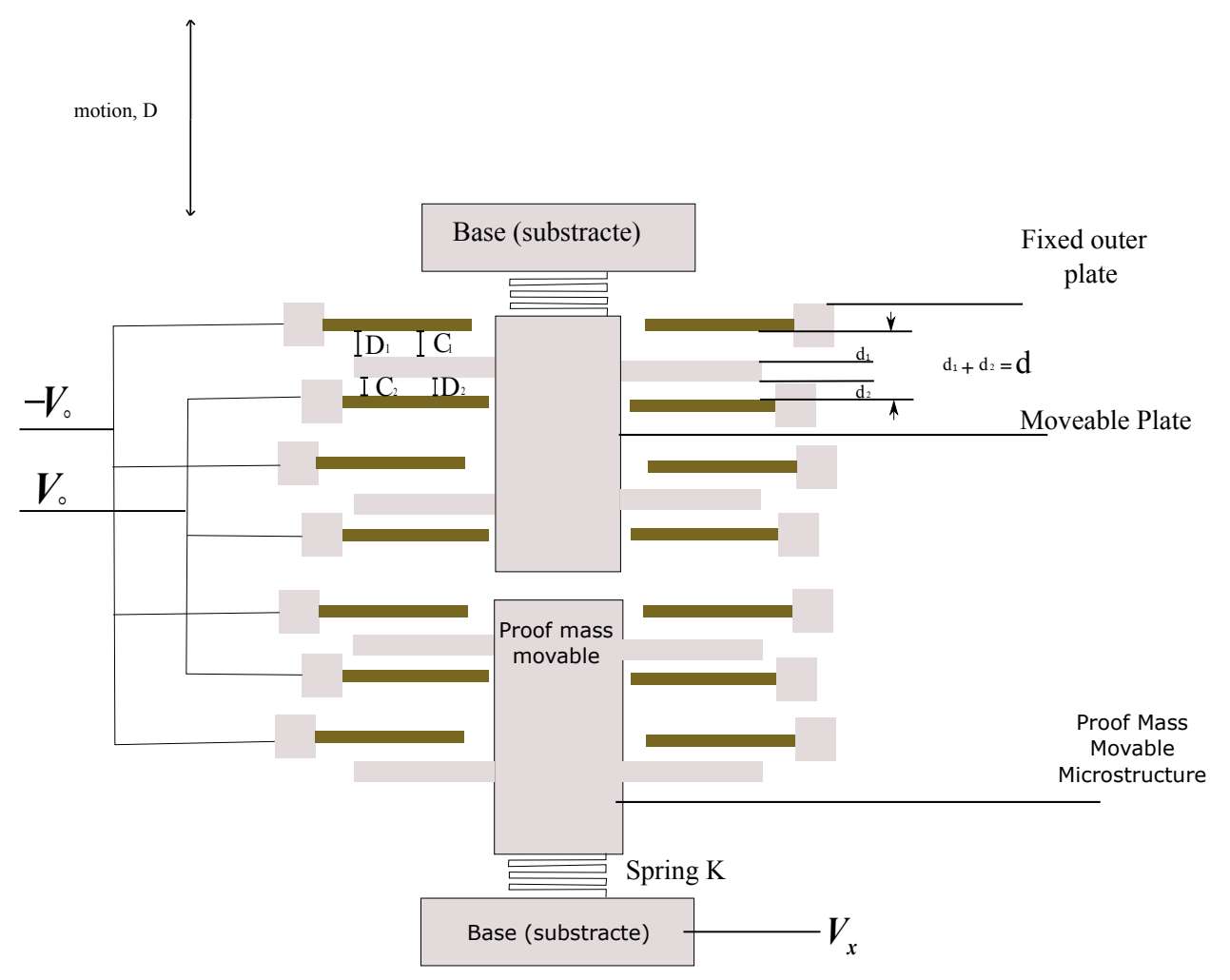

Figure 1.2: Electronic Circuit [2]

As shown in Fig. 1.2, each sensor has many capacitor sets. All upper capacitors are wired parallel to an overall capacitance $C_{1}$. Likewise, all lower ones are parallel to an overall capacitance $C_{2}$. Otherwise, the capacitance difference could be negligible 
to detect. Eq. 1.4 could be applied to all of the system. Fig. 1.2 shows a simplified electric circuit that measures the capacitance change. For example, Analog Devices accelerometer has 46 pairs of capacitors. The fixed plates of sensor are driven by $1 \mathrm{MHz}$ square waves with an amplitude voltage of a value of $V_{0}$ coming out of an oscillator. The voltage output $V_{x}$ is the voltage of the proof mass [9], as

$$
\begin{gathered}
\left(V_{x}+V_{0}\right) C_{1}+\left(V_{x}-V_{0}\right) C_{2}=0 \\
V_{x}=V_{0} \frac{C_{2}-C_{1}}{C_{2}+C_{1}}=\frac{D}{d} V_{0}
\end{gathered}
$$

where:

$C_{1}=$ upper cantilever beam capacitance.

$C_{2}=$ lower cantilever beam capacitance.

$V_{x}=$ voltage output.

$V_{0}=$ voltage input.

$D=$ motion

$d=$ the distance between the electrodes. 


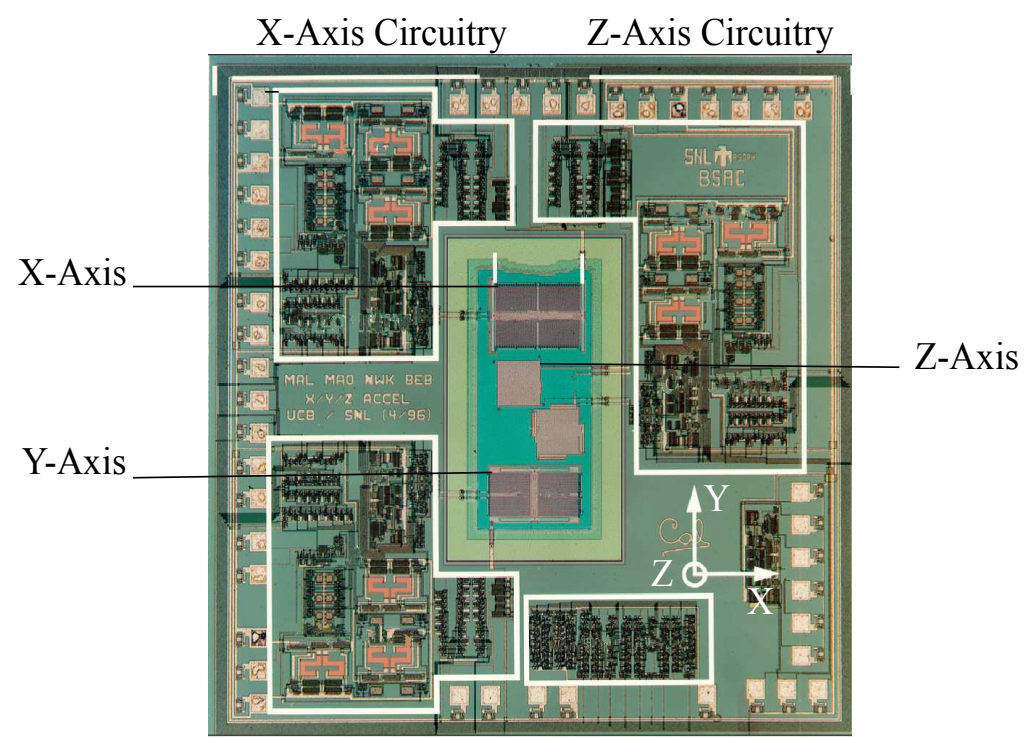

Y-Axis Circuitry Master Clock

Figure 1.3: 3D MEMS Accelerometer Packaging [3]

As shown in Fig. 1.3, there are many ways to package the 3D MEMS accelerometer. However, the most common ways are by separating each axis $\left(A_{x}, A_{y}\right.$ or $\left.A_{z}\right)$ from the other ones, or connecting them to the same proof mass [11].

The MEMS accelerometer sensor market grew from $\$ 700-800 \mathrm{M}$ in 2007 to $\$ 11,900$ M in 2015 [12]. Although a new technology has been employed to create MEMS sensors,sensor perfection is not guarantied. Sensor qualification is the last step of the manufacturing process to insure that the characteristics and performance of the sensors are in the specification range before shipping to the end user. The estimated costs of the MEMS accelerometer is about $1-2 \%$ to $40-50 \%$ of the total cost which include the cost of testing time [13]. Hence, the cost of the sensor can be reduced by cutting the testing time. The main purpose of this project is to develop a testing methodology and equipment to calibrate the MEMS accelerometer in three axes while reducing the testing time. 


\section{$1.2 \quad$ Scope}

The scope of this project is to develop a test methodology and equipment for mechanical testing of 3D-axes accelerometers. The testing system is designed to be able to test any accelerometer in 3D axes within this range starting from a minimum acceleration $0.1 \mathrm{~g}$ to the maximum acceleration $50 \mathrm{~g}$. The system will require high resolution for the encoder. The system has used absolute encoder 19 bits resolution and 524288 lines/rev. As a result, the equipment used to acquire the values of acceleration can detect an angular velocity as low as $0.0144^{\circ} / \mathrm{s}$ and will also be able to test a large range of accelerations in a short time in conjugation with a DC motor with a high torque of 18 in.lbs. and maximum speed of 1750 RPM. The proposed system tests multiple axes simultaneously for a large range of g ranging up to $50 \mathrm{~g}$. Moreover, the test system has to be designed to meet the requirement of providing highly accurate rotational motion, compact and reliable. 


\subsection{Outline of Study}

Chapter 1 includes a definition of the accelerometer and explains the principle of working of the accelerometer.

Chapter 2 includes a definition of the error that accompanies MEMS accelerometer sensors. Moreover, This chapter includes the two ways methodologies to qualify the sensor performance and determine the sensor errors; The first one used for single axis (Linear Regression Technique), and another one is 3D-axes testing scheme relying on the Kalman filter which is developed for 3-axis test.

Chapter 3 discusses and explains all equipment that is used to develop the system and it includes (hardware and software). The system was designed to generate rotation motion up to 844 RPM for the sensor test to get 50 g acceleration. Fully 3-D axes testing system was studied including dynamic and kinematic modeling. In Chapter 4, the testing result and error analysis for 3D axes MEMS accelerometer are discussed.

In Chapter 5, the summary, conclusion, and future works are presented. 


\section{Chapter 2}

\section{Testing Methodology and Error Analysis of MEMS Accelerometer}

Although the MEMS Industry's sensors are very accurate, errors such as bias offsets, scale factor errors, and misalignment could still be further improved. The testing methodology has to be developed to validate the sensor performance and determine all the sensor errors described above while reducing the testing time. IEEE recommends practice for precision centrifuge testing of linear acceleration and describes the general testing procedure for inertial sensors like MEMS accelerometers [14]. The main principle behind testing accelerometers is to expose an accelerometer to a known acceleration and to compare the output of the sensor with the input in a way that satisfies the design requirements. In this study, the input acceleration comes from a rotary motion. An acceleration sensor rotating about a fixed axis experiences two different accelerations; tangential and normal acceleration. These accelerations are related to the distance from the axis of rotation and angular velocity. Fig. 2.1 illustrates the scheme of acceleration input for the test setup. By varying the angular velocity and distance from the axis of rotation, it is possible to expose an accelerom-

eter to a desired acceleration value. The accelerometer can be oriented so that the desired acceleration stimulates one, two, or all three axes of the sensor. 


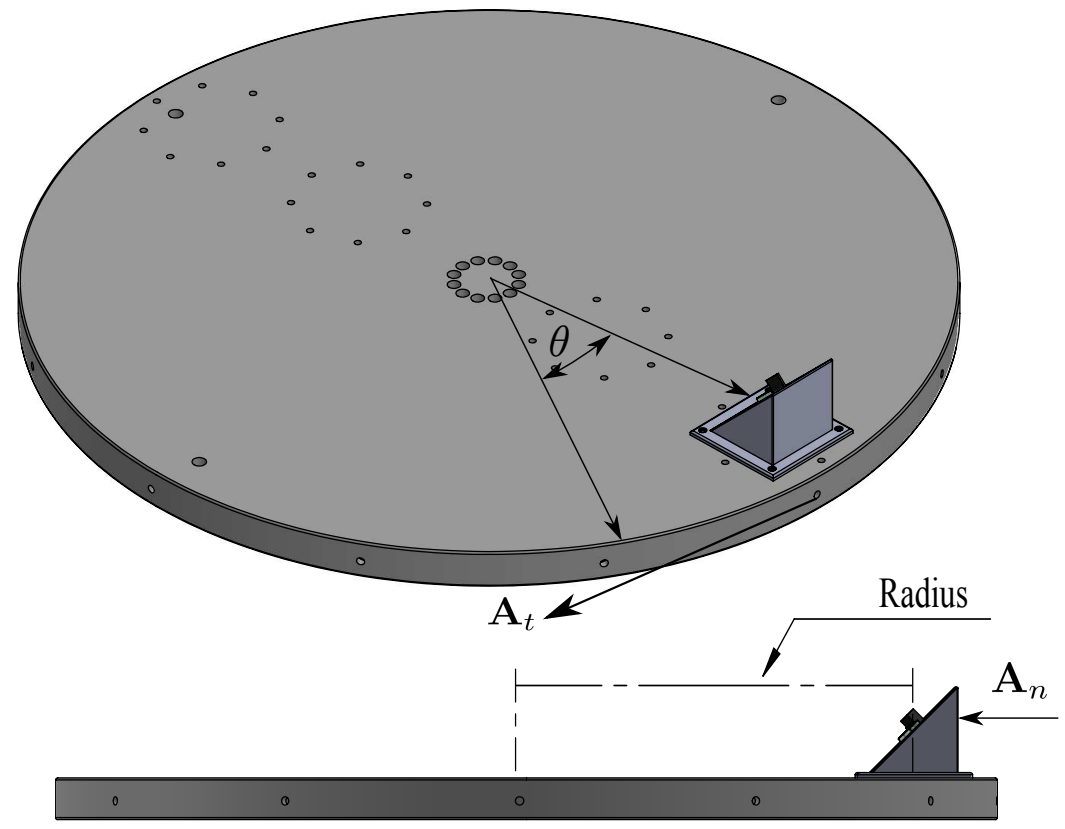

Figure 2.1: Scheme of Testing Accelerometer

where:

$\mathbf{R}=$ radius.

$\mathbf{A}_{n}=$ normal acceleration.

$\mathbf{A}_{t}=$ tangential acceleration.

$\boldsymbol{\theta}=$ the angular displacement. 


\subsection{Generation of Acceleration Input}

\subsubsection{Single Axis Test Mode}

\subsubsection{General}

Fig. 2.1 shows that the rate table is used to generate the acceleration input for sensor calibration. The accelerations caused by the circular motion of rate table are expressed by the following equations [15].

$$
\nu=\omega r
$$

$$
\begin{aligned}
& \mathbf{v}=\frac{d \boldsymbol{\theta}}{d t} \times \mathbf{r}=\boldsymbol{\omega} \times \mathbf{r} \\
& a_{t}=\alpha r, \quad a_{n}=\omega^{2} r \\
& \mathbf{a}=\boldsymbol{\alpha} \times \mathbf{r}+\boldsymbol{\omega} \times(\boldsymbol{\omega} \times \mathbf{r})
\end{aligned}
$$

where:

$\boldsymbol{\omega}=$ the angular velocity.

$\mathbf{v}=$ linear velocity.

$\mathbf{r}=$ distance from the center of rate table to sensor origin.

$t=$ time.

$d \boldsymbol{\theta}=$ the angular displacement.

$\boldsymbol{\alpha}=$ angular acceleration .

$\mathbf{a}=$ total acceleration. 


$$
\begin{aligned}
& a_{t}=\text { tangential acceleration. } \\
& a_{n}=\text { centripetal acceleration. }
\end{aligned}
$$

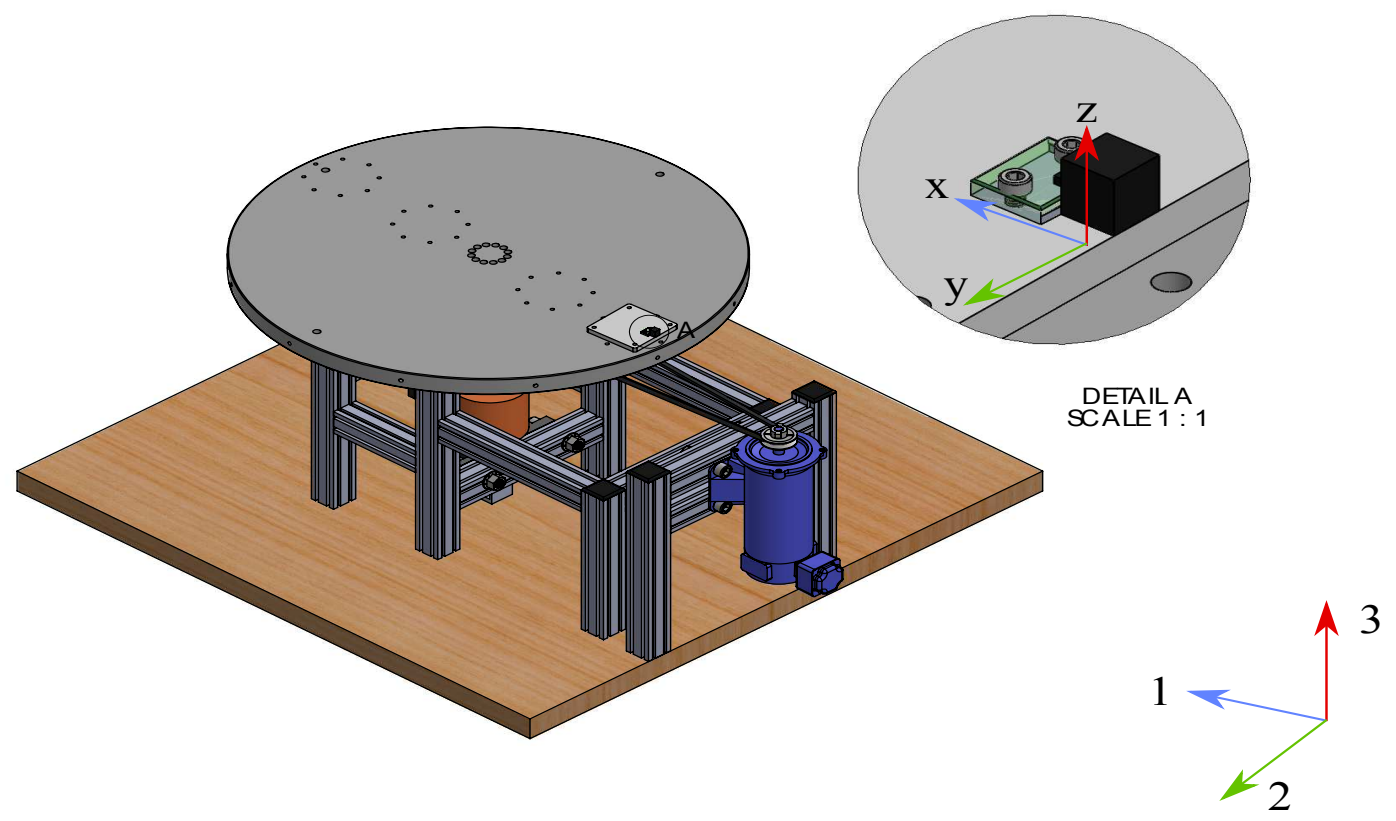

Figure 2.2: Single Axis Test about $x$ Axis

As shown in the Fig. 2.2, to expose only one axis of the accelerometer to the desired acceleration, the axis of interest $(x)$, should be oriented in the direction of the normal acceleration of the rotary motion. Fig. 2.2 shows the $x$-axis of the sensor oriented toward the center of the rotary motion; the $y$-axis is oriented towards the tangential acceleration (tangential acceleration is set to be zero for this study because the change in angular velocity to the time is very small), and the $z$-axis is oriented in the vertical direction. 


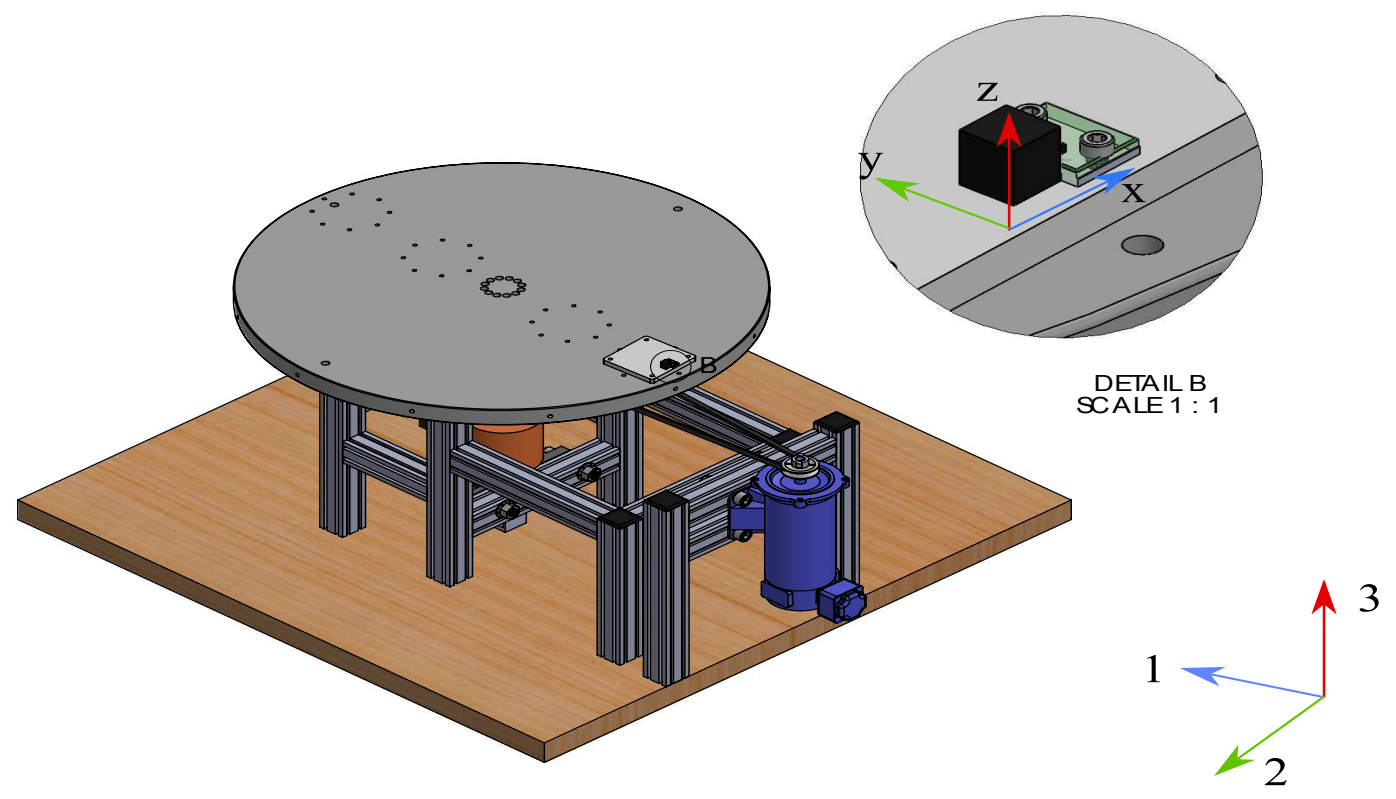

Figure 2.3: Single Axis Test about $y$ Axis

As shown in Fig. 2.3 to expose only one axis of the accelerometer to the desired acceleration, the axis of interest $(y)$ should be oriented in the direction of the normal acceleration of the rotary motion. Fig. 2.3 shows the $y$-axis of the sensor oriented toward the center of the rotary motion; the $x$-axis is oriented towards the tangential acceleration, and the $z$-axis is oriented in the vertical direction. 


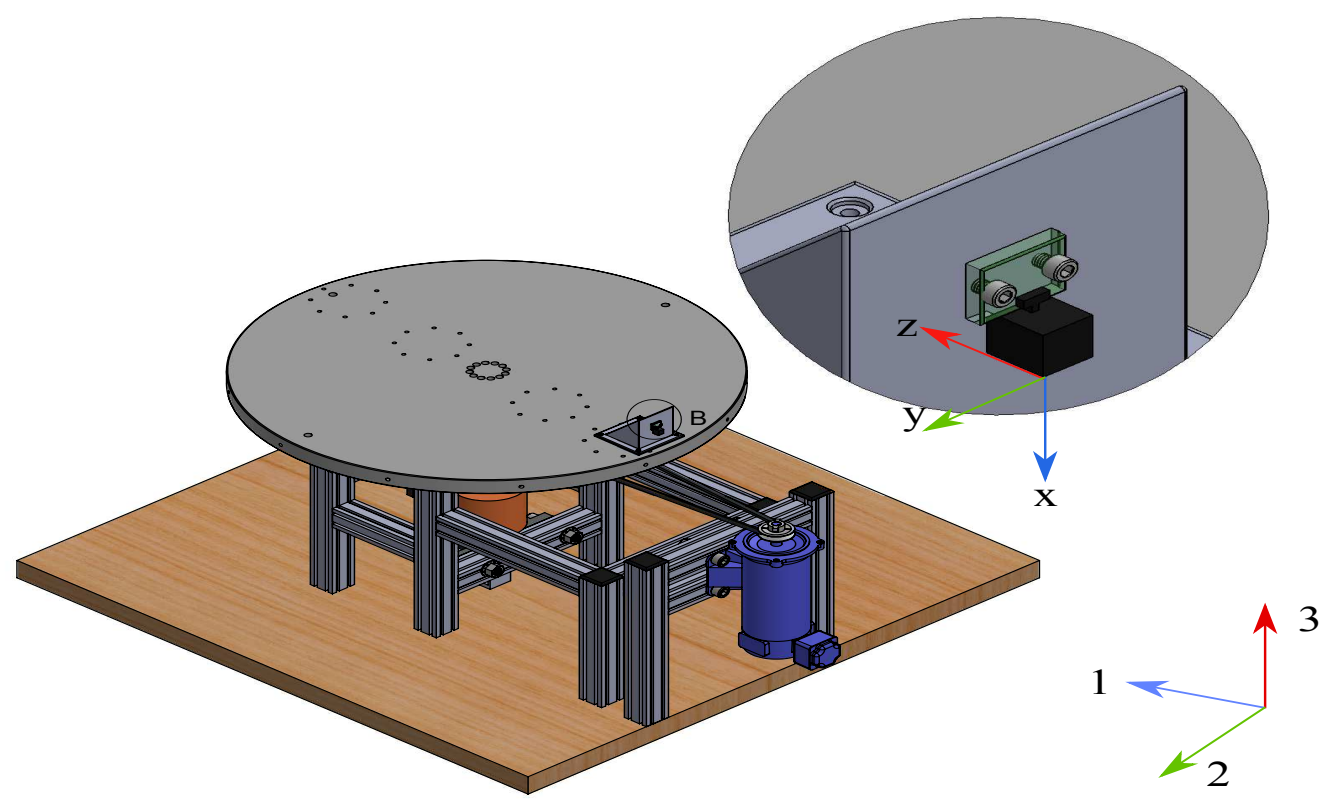

Figure 2.4: Single Axis Test about $z$ Axis

Fig. 2.4 shows that to expose only one axis of the accelerometer to the desired acceleration, the axis of interest $(z)$ should be oriented in the direction of the normal acceleration of the rotary motion. As shown in Fig. 2.4, to achieve $z$ orientation, it needs to use $45^{\circ}$ block. Fig. 2.4 shows the z-axis of the sensor oriented toward the center of the rotary motion; the $y$-axis is oriented towards the tangential acceleration, and the $x$-axis is oriented in the vertical direction. 


\subsubsection{Error Model of MEMS Accelerometer Sensor}

In the case of single axis testing, to perform an accelerometer sensor test, an accelerometer error model must be constructed, and the coefficients in the error equations must be determined. The model representing the relationship between the measured input and the output of the sensor is given by Eq. (2.3) [16].

$$
a_{i}^{*}=\left(1+\varepsilon_{i}\right) a_{i}-\beta_{i}-\vartheta
$$

where:

$a_{i}^{*}=$ output acceleration along the $i^{t h}$-axis of accelerometer.

$a_{i}=$ input acceleration along the $i^{t h}$-axis.

$\varepsilon_{i}=$ scale factor error of the $i^{t h}$-axis of accelerometer.

$\beta_{i}=$ the bias offset of the sensor along its $i^{t h}$-axis.

$\vartheta=$ random error with an average of zero.

$i=1,2$ and 3 axis.

$*=$ output acceleration from accelerometer sensor.

By equating the coefficients from Eq. (2.3), the scale factor and bias can be expressed as:

$$
\begin{gathered}
1+\varepsilon_{i}=\alpha \\
\beta_{i}=\beta
\end{gathered}
$$

This general equation is used to process data and produce results with error information about the tested accelerometer. Suppose we have $n$ pairs of data collected 
$\left(a_{i}^{*}, a_{i}\right),\left(a_{i}^{*}, a_{i}\right) \ldots\left(a_{i}^{*}, a_{i}\right)$. A scatter plot of $a_{i}^{*}$ vs. $a_{i}$ can be generated. An estimated regression line can lead to the calculation of scale factor and bias. The best fit to the data is expected to be in the form of Eq. (2.6).

$$
a_{i}^{*}=\alpha a_{i}-\beta-\vartheta
$$

where:

$$
\begin{aligned}
& \alpha=\text { scale factor error }+1 . \\
& \beta=\text { the bias offset of the sensor. }
\end{aligned}
$$

The random error $\vartheta$ can be ignored because a mean value of the random error is zero. Therefor, Eq. (2.6) can be reduced to

$$
a_{i}^{*}=\alpha a_{i}-\beta
$$

The same method can be used to determine the misalignment of an axis about another axis. Again $\mathrm{n}$ data points are required to find the misalignment of an axis with respect to an orthogonal reference axis. The general equation relating one axis to a reference axis is shown in Eq. (2.8).

$$
a_{j}^{*}=\theta_{i j} a_{i}-\beta_{i}
$$

where:

$\theta_{i j}=$ the coefficient of misalignment along the $i^{t h}$-axis in reference to the $j^{t h}$-axis. $\beta_{i}=$ the bias offset of the sensor along its $i^{t h}$-axis. 
The main disadvantages of the single axis test are; i) According to the reference [14] it would take a long time to install the parts and to collect the data for nine positions. ii)In order to test each position for 3D accelerometer sensor after each position test the sensor needs to reorient the axis of the sensor to test another position, and this process maybe lead to cause errors in the angle and alignment of the sensor. Moreover, in the single axis test, each axis is needed to be tested individually with other axes to find the errors (Scale Factor, Bias, and Misalignment).

The single axis test uses the least square fit method to determine the errors. Suppose that we have $n$ pairs of observations. For example, if the collected data is $\left\{\left(x_{1}, y_{1}\right)\right.$. . . $\left.\left(x_{n}, y_{n}\right)\right\}$, so the define of the errors will be associated with $a_{i}^{*}=\alpha a_{i}-\beta$ as shown in Fig. 2.5.

where:

$$
\begin{aligned}
& a_{i}^{*}=\text { output acceleration along the } i^{\text {th }} \text { axis of accelerometer. } \\
& a_{i}=\text { input acceleration along the } i^{\text {th }} \text { axis. } \\
& \alpha=\text { scale factor error. } \\
& \beta=\text { the bias offset of the sensor. }
\end{aligned}
$$




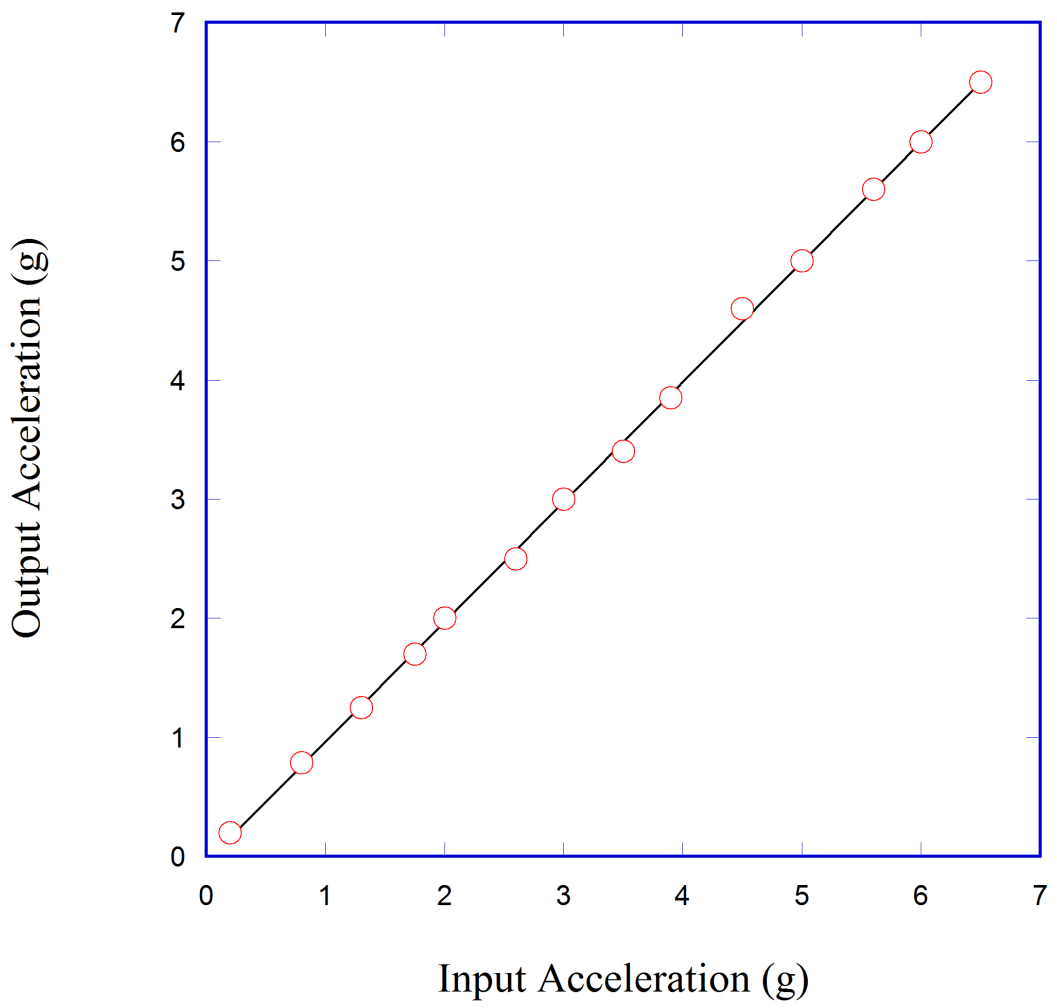

Figure 2.5: Output vs. Input Acceleration Along $i$-Axis

The plot depicted in Fig. 2.5 shows a comparison between the input and output acceleration values along the $i$-axis. The best fit to the data leads to the estimation of the scale factor and bias associated with the $i$-axis [17]. The collected data in a single axis test is also used to determine the misalignment associated with the other two axes in reference to the axis of interest. For example, from the $i$-axis test mentioned above, it is possible to measure the misalignment associated with the $j$ axis in reference to our ideal $i$-axis $\left(\theta_{i j}\right)$ and the misalignment associated with the $k$-axis in reference to our ideal $i$-axis $\left(\theta_{i k}\right)$ as shown in Fig. 2.6. 


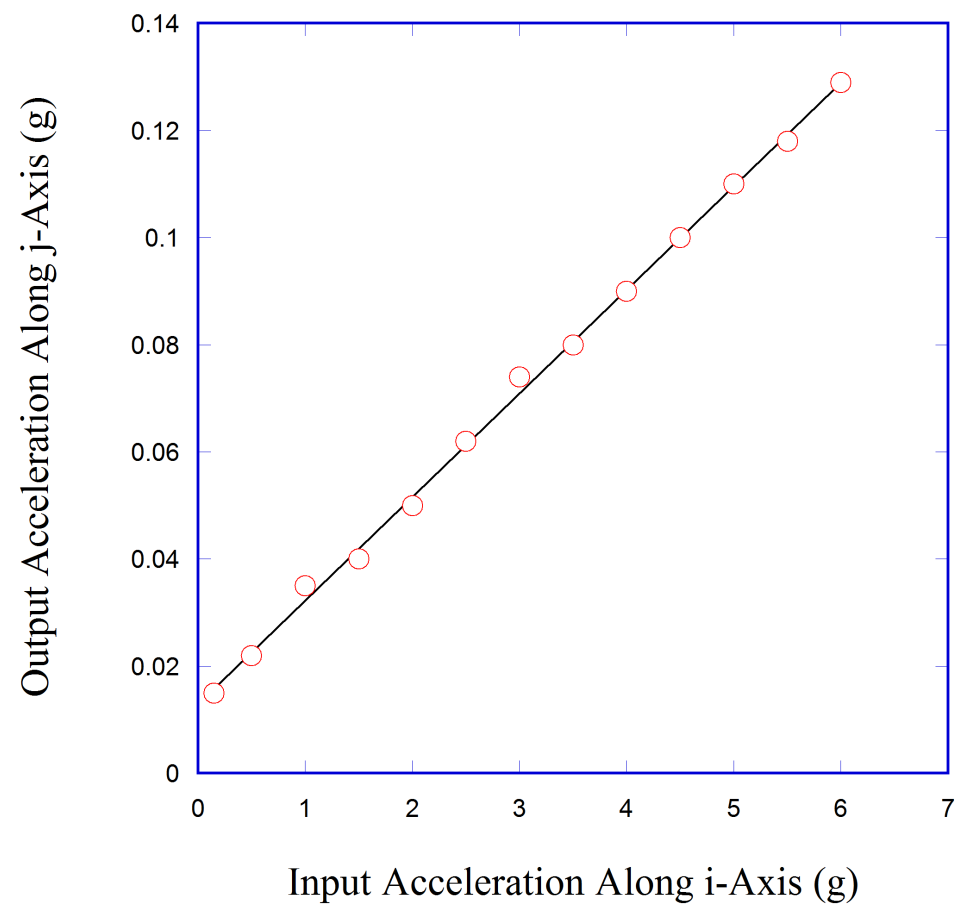

Figure 2.6: Output Acceleration Along $j$-Axis vs. Input Acceleration Along $i$-Axis

The misalignment angles $(\theta)$ associated with the $j$-axis and the $k$-axis in reference to the $i$-axis are measured form the following expressions.

$$
\begin{aligned}
& a_{j}^{*}=\theta_{i j} a_{i}-\beta_{i} \\
& a_{k}^{*}=\theta_{i k} a_{i}-\beta_{i}
\end{aligned}
$$

where:

$\theta i j=$ angle of misalignment of the $j$-axis with respect to the reference $i$-axis. $\theta i k=$ angle of misalignment of the $k$-axis with respect to the reference $i$-axis. 


\subsection{The Least Square Method Equation}

Suppose that we have $\mathrm{n}$ pairs of observations $\left\{y_{1}-\left(a x_{1}+b\right) . . y_{n^{-}}\left(a x_{N}+b\right)\right\}$. A typical scatter plot of this observed data and a candidate for the estimated regression line are shown in Fig. 2.7. The estimated values of the parameters $a$ and $b$ in the observed data should result in a line that is a "best fit" to the data. The German scientist Karl Gauss (1777-1855) proposed an estimation of the parameters $a$ and $b$ to minimize the sum of the squares of the vertical deviations as shown in Fig. 2.7. Estimating the regression parameters is commonly called the Method of Least Squares [4].

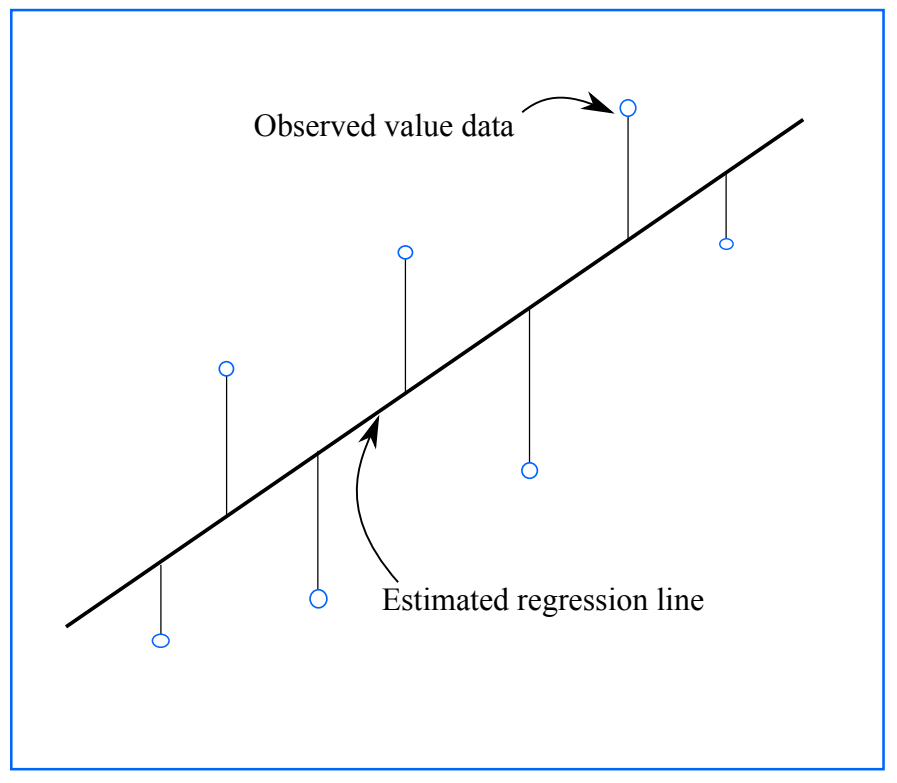

Figure 2.7: Deviations of the Data From the Estimated Regression Model [4] 
The sum of the square of the deviations of the observations from the true regression line is:

$$
\begin{gathered}
E(a, b)=\sum_{n=1}^{N}\left(y_{n}-\left(a x_{n}+b\right)\right)^{2} \\
\frac{\partial E}{\partial a}=0, \quad \frac{\partial E}{\partial b}=0 \\
\frac{\partial E}{\partial a}=\sum_{n=1}^{N} 2\left(y_{n}-\left(a x_{n}+b\right)\right)\left(-x_{n}\right)=0 \\
\frac{\partial E}{\partial b}=\sum_{n=1}^{N} 2\left(y_{n}-\left(a x_{n}+b\right)\right)(1)=0
\end{gathered}
$$

Rewrite these equations as

$$
\begin{aligned}
\left(\sum_{n=1}^{N} x_{\mathrm{n}}^{2}\right) a+\left(\sum_{n=1}^{N} x_{n}\right) b & =\sum_{n=1}^{N} x_{n} y_{n} \\
\left(\sum_{n=1}^{N} x_{\mathrm{n}}\right) a+\left(\sum_{n=1}^{N} 1\right) b & =\sum_{n=1}^{N} y_{n}
\end{aligned}
$$


Matrix equation will be:

$$
\left(\begin{array}{ccc}
\sum_{n=1}^{N} x_{n}^{2} & \sum_{n=1}^{N} x_{n} \\
\sum_{n=1}^{N} x_{n} & \sum_{n=1}^{N} 1
\end{array}\right)\left(\begin{array}{l}
a \\
b
\end{array}\right)=\left(\begin{array}{c}
\sum_{n=1}^{N} x_{n} y_{n} \\
\sum_{n=1}^{N} y_{n}
\end{array}\right)
$$

The matrix is invertible, which implies

$$
\left(\begin{array}{l}
a \\
b
\end{array}\right)=\left(\begin{array}{cc}
\sum_{n=1}^{N} x_{n}^{2} & \sum_{n=1}^{N} x_{n} \\
\sum_{n=1}^{N} x_{n} & \sum_{n=1}^{N} 1
\end{array}\right)^{-1}\left(\begin{array}{c}
\sum_{n=1}^{N} x_{n} y_{n} \\
\sum_{n=1}^{N} y_{n}
\end{array}\right)
$$

Denote the matrix $M$ is

$$
/ M /=\left(\begin{array}{cc}
\sum_{n=1}^{N} x_{n}^{2} & \sum_{n=1}^{N} x_{n} \\
\sum_{n=1}^{N} x_{n} & \sum_{n=1}^{N} 1
\end{array}\right)
$$

The determinant of $M$ is

$$
/ M /=\sum_{n=1}^{N} x_{n}^{2} \sum_{n=1}^{N} 1-\sum_{n=1}^{N} x_{n} \sum_{n=1}^{N} x_{n}
$$

as

$$
\bar{x}=\frac{1}{N} \sum_{n=1}^{N} x_{n}
$$


SO

$$
\begin{aligned}
& \mid M /=\sum_{n=1}^{N} x_{n}^{2}-(N \bar{x})^{2} \\
& =N^{2}\left(\frac{1}{N} \sum_{n=1}^{N} x_{n}^{2}-\bar{x}^{2}\right) \\
& =N^{2} \frac{1}{N} \sum_{n=1}^{N}\left(x_{n}-\bar{x}\right)^{2}
\end{aligned}
$$

By using the definition of variance, we notice the above could also be written as

$$
\begin{gathered}
/ M /=N^{2} \sigma_{x}^{2} \\
\left(\begin{array}{l}
a \\
b
\end{array}\right)=\frac{1}{N^{2} \sigma_{x}^{2}}\left(\begin{array}{cc}
N & -N \bar{x} \\
-N \bar{x} & \sum_{n=1}^{\mathrm{N}} x_{n}^{2}
\end{array}\right)^{-1}\left(\begin{array}{c}
\sum_{n=1}^{N} x_{n} y_{n} \\
\sum_{n=1}^{N} y_{n}
\end{array}\right) \\
a=\frac{N \sum_{n=1}^{N} x_{n} y_{n}-N \bar{x} \sum_{n=1}^{\mathrm{N}} y_{n}}{N^{2} \sigma_{x}^{2}}
\end{gathered}
$$




$$
b=\frac{-N \sum_{n=1}^{N} x_{n} y_{n}+\sum_{n=1}^{N} x_{n}^{2} \sum_{n=1}^{N} y_{n}}{N^{2} \sigma_{x}^{2}}
$$

$$
\begin{gathered}
\bar{x}=\frac{1}{N} \sum_{n=1}^{N} x_{i} \\
\sigma_{x}^{2}=\frac{1}{N} \sum_{n=1}^{N}\left(x_{i}-\bar{x}\right)^{2}
\end{gathered}
$$

Therefore, there is another expression for

$$
\begin{gathered}
a=\frac{\sum_{n=1}^{N} 1 \sum_{n=1}^{N} x_{n} y_{n}-\sum_{n=1}^{N} x_{n} \sum_{n=1}^{N} y_{n}}{\sum_{n=1}^{N} 1 \sum_{n=1}^{N} x_{n}^{2}-\sum_{n=1}^{N} x_{n} \sum_{n=1}^{N} x_{n}} \\
b=\frac{\sum_{n=1}^{N} x_{n} \sum_{n=1}^{N} x_{n} y_{n}-\sum_{n=1}^{N} x_{n}^{2} \sum_{n=1}^{N} y_{n}}{\sum_{n=1}^{N} x_{n} \sum_{n=1}^{N} x_{n}-\sum_{n=1}^{N} x_{n}^{2} \sum_{n=1}^{N} 1}
\end{gathered}
$$




\subsubsection{Tri-Axis Test Mode}

\subsubsection{General}

A rate table is used to generate the acceleration, but in this situation, the input acceleration needs to be analyzed to three components 1,2 , and 3 . Therefore, in order to test the three axes of the sensor at once, the orientation of the sensor has to be changed so that the acceleration generated by the rate table and the gravity acceleration are distributed equally on the three axes. As shown in Fig. 2.8, to simplify the design, rotational angle $\phi$ about 3 -axis is fixed at $45^{\circ}$ so that the acceleration $A_{x}$ and $A_{y}$ on the mounting block are the same. Then, the rotational angle $\psi$ about 1 -axis is varied until $\mathrm{A}_{x}, \mathrm{~A}_{y}$, and $\mathrm{A}_{z}$ on the mounting block are equal [7].

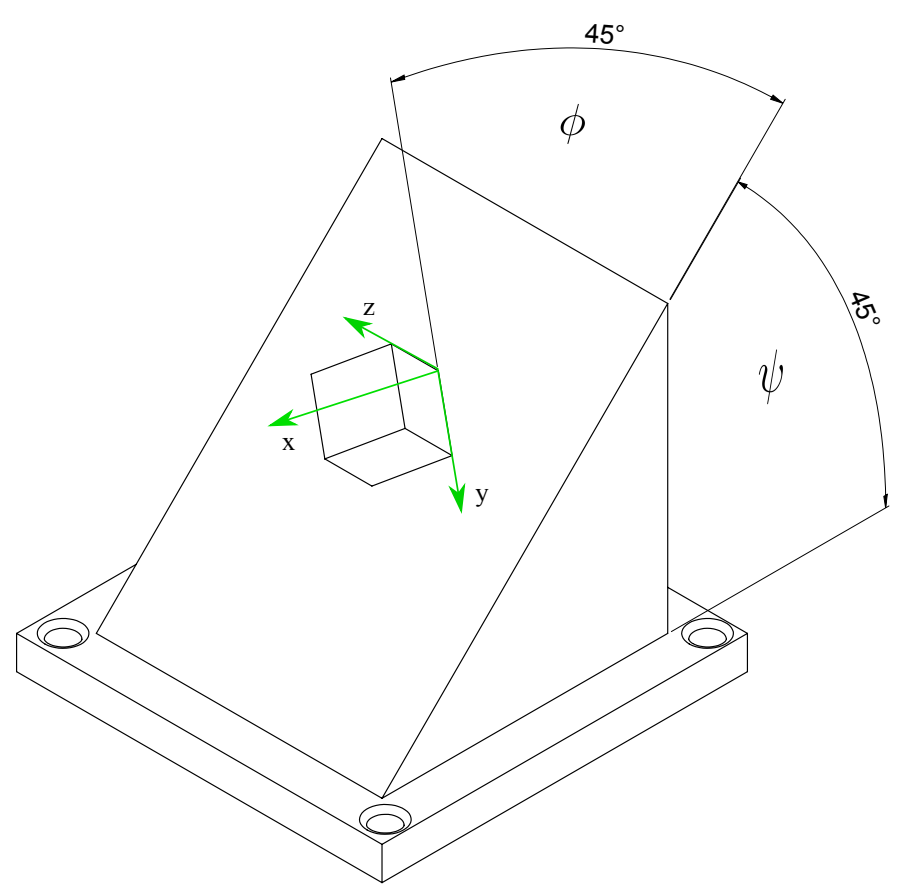

Figure 2.8: Position of the Sensor for Tri Axes Test 
There are two approaches to limit the amount of the acceleration that will be generated during circular motion on the turntable. For the first approach, the sensor is mounted at the center of the $45^{\circ}$-mounting block. The mounting block is placed on the rate table at a distance of $R$ from the center of the table as shown in Fig. 2.9. For the second approach, the angular rate can be controlled by using a speed controller. The speed controller is used to control the value of the voltage that is needed to rotate a DC motor at a specific speed. The testing system used in the current study was designed to test a sensor that has a lower/upper range of \pm 50 g. Therefore, the distance from sensor origin to the center of the center of the rate table $(R)$ should be $0.45 \mathrm{~m}$, and the angular velocity of the rate table should be 844 RPM. However, the available ADI sensor at the time of the current study had a range of $\pm 18 \mathrm{~g}$. Thus, the theoretical value of the angular velocity of the rate table should be 304 RPM with the same value of $R$. Moreover, in this case, each axis of the mounting block coordinate system reciprocally aligns with the axes of the rate table coordinate system. consequently, 1-axis is in the tangential direction, 2 -axis is in the radial direction, and 3-axis points up. 


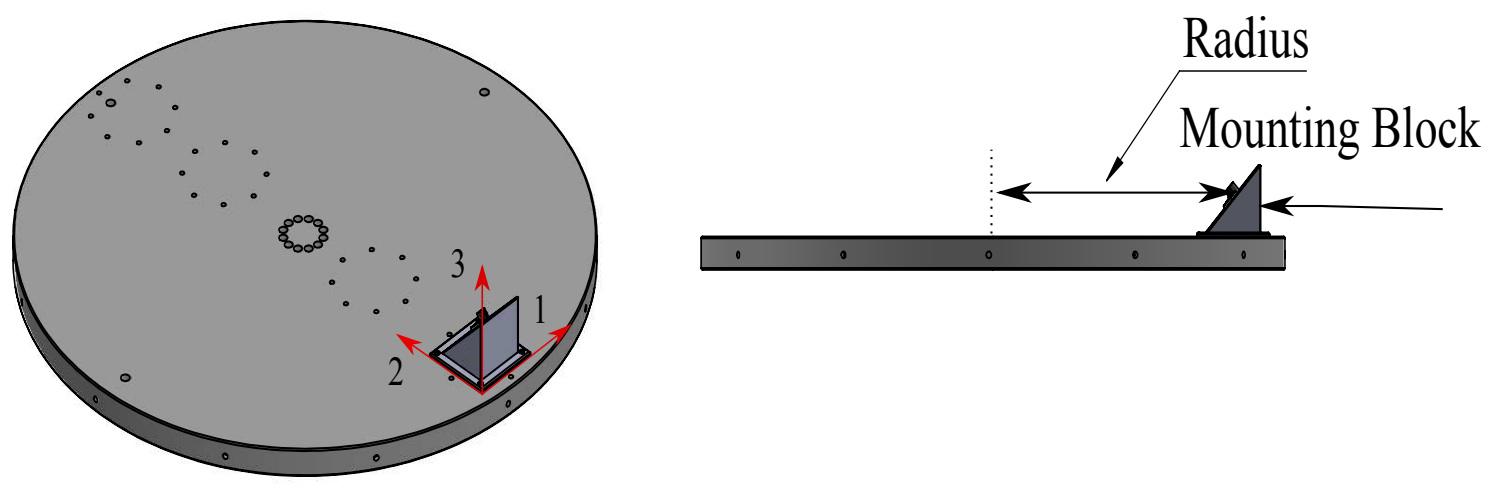

Figure 2.9: Scheme of Testing 3D-Axes Accelerometer

\subsubsection{Transfer Coordinates}

The transformation from the global coordinates system of the measured input to the local coordinates system of the sensor output is considered the first step for measuring the error by the multi-axis test. The global and local coordinate represented by red and green coordinates symbols,respectively in Fig. 2.10. The transformation of coordinate is used to make comparison between the input and output on the same coordinate system. The transformation of coordinates is shown in Fig. 2.10, and it could be expressed as following: 


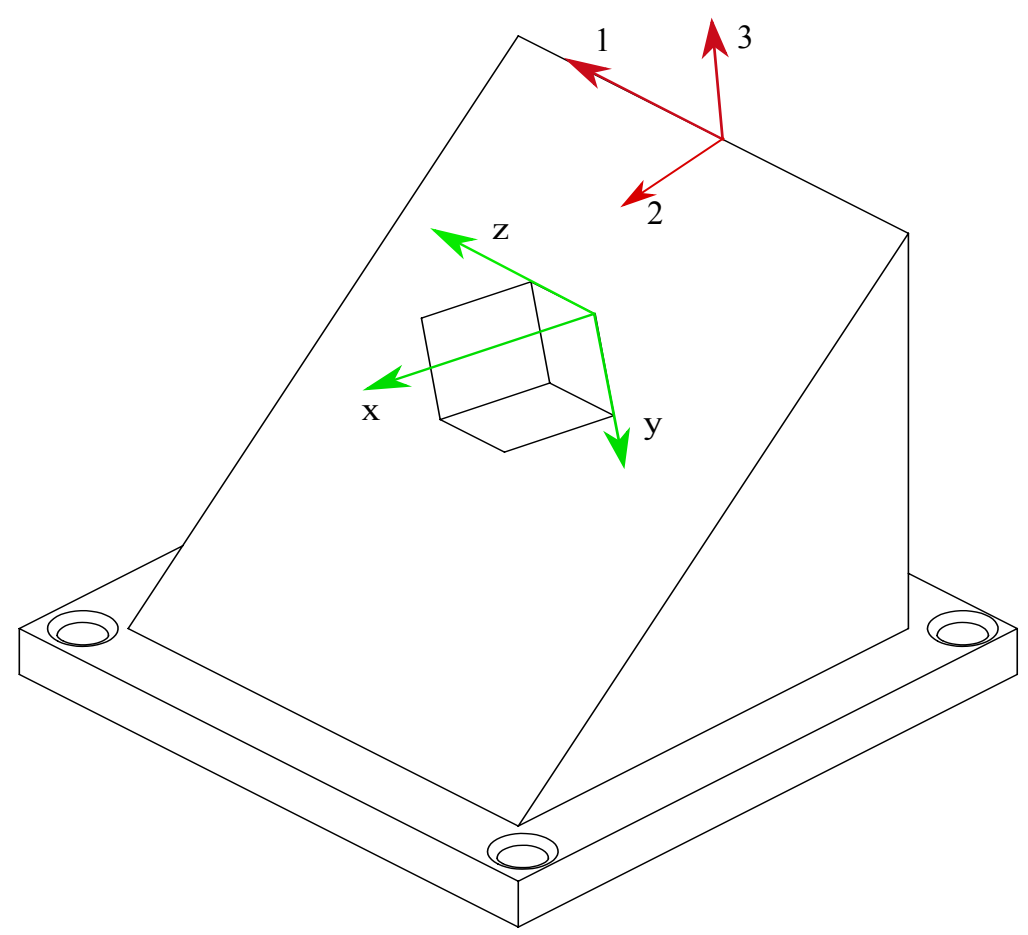

Figure 2.10: Transform Global Coordinates to the Local Coordinates

The orthogonal triad of unit vectors parallel to the inertial axes $X, Y$, and $Z$ are $\hat{\mathbf{I}}, \hat{\mathbf{J}}$ and $\hat{\mathbf{K}}$ respectively.

$$
\mathbf{A}=A_{1} \hat{\mathbf{I}}+A_{2} \hat{\mathbf{J}}+A_{3} \hat{\mathbf{K}}
$$

where:

$$
\begin{aligned}
& A_{1}=\text { tangential acceleration. } \\
& A_{2}=\text { normal acceleration. } \\
& A_{3}=\text { gravitational acceleration }(\mathrm{g}) .
\end{aligned}
$$

$$
\{\mathbf{A}\}_{\text {new }}=[\mathbf{T}]\{\mathbf{A}\}
$$

where:

$$
[\mathbf{T}]=\text { the transformation matrix. }
$$




$$
\begin{gathered}
\{\mathbf{A}\}_{\text {new }}=[\mathbf{T}]\left\{\mathbf{A}_{1}, \mathbf{A}_{2}, \mathbf{A}_{3}\right\} \\
{[\mathbf{T}]=\left[\mathbf{R}_{1}\right]\left[\mathbf{R}_{2}\right]\left[\mathbf{R}_{3}\right]} \\
{[\mathbf{T}]=\left[\begin{array}{ccc}
\sin (\psi) \sin (\phi) \sin (\theta)+\cos (\theta) \cos (\phi), & \cos (\psi) \sin (\phi), & \sin (\psi) \cos (\theta) \sin (\phi)-\sin (\theta) \cos (\phi) \\
\sin (\psi) \sin (\theta) \cos (\phi)-\cos (\theta) \sin (\phi), & \cos (\psi) \cos (\phi), & \sin (\psi) \cos (\theta) \cos (\phi)+\sin (\theta) \sin (\phi) \\
\cos (\psi) \sin (\theta), & -\sin (\psi), & \cos (\psi) \cos (\theta)
\end{array}\right]}
\end{gathered}
$$

See Figure 3.6. 
where:

$\psi=$ rotation angle around 1 -axis.

$\theta=$ rotation angle around 2 -axis.

$\phi=$ rotation angle around 3 -axis.

$\mathbf{R}_{1}=$ the rotational matrices around the 1-axis.

$\mathbf{R}_{2}=$ the rotational matrices around the 2-axis.

$\mathbf{R}_{3}=$ the rotational matrices around the 3 -axis.

Therefor, the measured acceleration as result of transfer coordinate for each axis can be expressed as;

$$
\begin{gathered}
a_{x}^{*}=a_{n} \sin (\phi) \cos (\psi)-g \sin (\phi) \sin (\psi) \\
a_{y}^{*}=a_{n} \cos (\phi) \cos (\psi)-g \sin (\psi) \cos (\phi) \\
a_{z}^{*}=-a_{n} \sin (\psi)-g \cos (\psi)
\end{gathered}
$$

where:

$a_{x}^{*}=$ output acceleration along the $x$-axis.

$a_{n}=$ centripetal acceleration.

$a_{y}^{*}=$ output acceleration along the $y$-axis.

$a_{z}^{*}=$ output acceleration along the $z$-axis.

$\phi=$ rotation angle around 3 -axis.

$\psi=$ rotation angle around 1-axis.

$g=$ gravity

$*=$ input acceleration from encoder. 


\subsubsection{Kalman Filter}

The Kalman filter has been considered as the optimal solution to many tracking and data prediction tasks. Its used in the analysis of visual motion that has been frequently documented. The filter is constructed as a mean squared error minimizer. The purpose of the filter is to extract the required information from a signal and ignoring everything else [18]. The Kalman filter addresses the general problem of estimating the state measurement $\mathbf{x} \in \mathbf{R}_{n}$ of a discrete-time controlled process that is governed by the linear stochastic difference equation [19].

$$
\mathbf{x}_{k}=\mathbf{A} \mathbf{x}_{k-1}+\mathbf{B u}_{k-1}+\mathbf{w}_{k-1}
$$

where:

$\mathbf{x}_{k}=$ the state vector of the process at time $k$.

$\mathbf{A}=$ the state transition matrix of the process from the state at $k$ - 1 to the state at $k$.

$\mathbf{w}_{k}=$ the associated white noise process with known covariance.

Observations on this variable can be modelled in the form of

$$
\mathbf{z}_{k}=\mathbf{H x}_{k}+\mathbf{v}_{k}
$$

where:

$\mathbf{z}_{k}=$ the actual measurement of $\mathbf{x}$ at time $k$.

$\mathbf{H}=$ the noiseless connection between the state vector and the measurement vector.

$\mathbf{v}_{k}=$ the associated measurement error. 
The process noise covariance $\boldsymbol{Q}$ and measurement noise covariance $\mathbf{R}$ matrices are assumed stationary over time and are given by

$$
\begin{aligned}
& \mathbf{Q}=\mathbf{E}\left[\mathbf{w}_{k} \mathbf{w}_{k}^{T}\right] \\
& \mathbf{R}=\mathbf{E}\left[\mathbf{v}_{k} \mathbf{v}_{k}^{T}\right]
\end{aligned}
$$

where:

$$
\begin{aligned}
& \mathbf{Q}=\text { Process Noise Covariance. } \\
& \mathbf{R}=\text { Measurement Noise Covariance. }
\end{aligned}
$$

The mean squared error is:

$$
\mathbf{E}\left[\mathbf{e}_{k} \mathbf{e}_{k}^{T}\right]=\mathbf{P}_{k}
$$

where:

$$
\begin{aligned}
& \mathbf{P}_{k}=\text { the error covariance matrix at time } k . \\
& \mathbf{E}=\text { expectation operator. } \\
& \mathbf{e}_{k}=\text { a posteriori estimate errors. }
\end{aligned}
$$

Eq. (2-41) may be expanded to give;

$$
\mathbf{P}_{k}=\mathbf{E}\left[\mathbf{e}_{k} \mathbf{e}_{k}^{T}\right]=\mathbf{E}\left[\left(\mathbf{x}_{k}-\hat{\mathbf{x}}_{k}\right)\left(\mathbf{x}_{k}-\hat{\mathbf{x}}_{k}\right)^{T}\right]
$$

Assuming the prior estimate of $\hat{\mathbf{x}}_{k}$ is called $\hat{\mathbf{x}}^{\prime}$, and was gained by knowledge of the system. It is possible to write an updated equation for the new estimate, combing the old estimate with measured data thus; 


$$
\hat{\mathbf{x}}_{k}=\hat{\mathbf{x}}_{k}^{\prime}+\mathbf{K}_{k}\left(\mathbf{z}_{k}-\mathbf{H} \hat{\mathbf{x}}_{k}^{\prime}\right)
$$

where:

$$
\begin{aligned}
& \mathbf{K}_{k}=\text { the Kalman gain. } \\
& \hat{\mathbf{x}}_{k}=\text { the posterior estimate. } \\
& \hat{\mathbf{x}}^{\prime}{ }_{k}=\text { the prior estimate. }
\end{aligned}
$$

$$
\mathbf{i}_{k}=\mathbf{z}_{k}-\mathbf{H}^{\prime}{ }_{k}
$$

At this point, it is noted that $\mathbf{x}_{k}-\hat{\mathbf{x}}_{k}{ }_{k}$ is the error of the prior estimate. The difference $\left(\mathbf{z}_{k}-\mathbf{H}_{\mathbf{x}^{\prime}}{ }_{k}\right)$ in Eq. (2.45) is called the measurement innovation, or the residual. The residual reflects the discrepancy between the predicted measurement $\mathbf{H}_{\mathbf{x}^{\prime}}{ }_{k}$ and the actual measurement $\mathbf{z}_{k}$. A residual of zero means that the measurement innovation are in complete agreement [19]. The $n \times m$ matrix $\mathbf{K}_{k}$ in Eq. (2.44) is chosen to be the gain or blending factor that minimizes the posteriori error covariance in Eq. (2.42). This minimization can be accomplished by first substituting Eq. (2.44) into the above definition of $\mathbf{e}_{k}$, substituting that into Eq. (2.42), performing the indicated expectations. Then by taking the derivative of the trace of the result with respect to $\mathbf{K}_{k}$ setting that result equal to zero, and then the $\mathbf{K}_{k}$ can be determined. One form of the resulting $\mathbf{K}_{k}$ that minimizes [5]. Eq. (2.42) is given by

$$
\mathbf{P}_{k}=\left(\mathbf{I}-\mathbf{K}_{k} \mathbf{H}\right) \mathbf{E}\left[\left(\mathbf{x}_{k}-\hat{\mathbf{x}}_{k}^{\prime}\right)\left(\mathbf{x}_{k}-\hat{\mathbf{x}}_{k}^{\prime}\right)^{T}\right]\left(\mathbf{I}-\mathbf{K}_{k} \mathbf{H}\right)-\mathbf{K}_{k} \mathbf{E}\left[\mathbf{v}_{k} \mathbf{v}_{k}^{T}\right] \mathbf{K}_{k}^{T}
$$

where:

$$
\mathbf{P}_{k}=\text { State Error Covariance Matrix. }
$$


$\mathbf{x}_{k}=$ System State Matrix.

$\mathbf{H}_{k}=$ Measurement transition Matrix.

Now solving for $\mathbf{K}_{k}$ gives;

$$
\mathbf{K}_{k}=\mathbf{P}_{k}^{\prime} \mathbf{H}^{T}\left(\mathbf{H P}_{k}^{\prime} \mathbf{H}^{T}+\mathbf{R}\right)^{-1}
$$

Looking at Eq. (2.47), as the measurement error covariance $\mathbf{R}$ approaches zero, the gain $\mathbf{K}_{k}$ weights the residual more heavily. Specifically,

$$
\lim _{\mathbf{R}_{k} \rightarrow 0} \mathbf{K}_{k}=\mathbf{H}^{-1}
$$

On the other hand, as the a priori estimate error covariance $\mathbf{P}_{k}^{\prime}$ approaches zero, the gain $\mathbf{K}_{k}$ weights the residual less heavily. Specifically

$$
\lim _{\mathbf{P}_{k}^{\prime} \rightarrow 0} \mathbf{K}_{k}=0
$$

Another way of thinking about the weighting by $\mathbf{K}$ is that as the measurement error covariance $\mathbf{R}$ approaches zero, the actual measurement $\mathbf{z}_{k}$ is trusted more and more, while the predicted measurement $\mathbf{H}_{\mathbf{x}^{\prime}}{ }_{k}$ is trusted less and less. On the other hand, as the a priori estimate error covariance $\mathbf{P}_{k}^{\prime}$ approaches zero the actual measurement $\mathbf{z}_{k}$ is trusted less and less, while the predicted measurement $\mathbf{H}_{\mathbf{x}^{\prime}}{ }_{k}$ is trusted more and more [5]. 


\subsubsection{The Discrete Kalman Filter Algorithm}

The Kalman filter estimates a process by using a form of feedback control. The filter estimates the process state at some time and then obtains feedback in the form of (noisy) measurements. The equations for the Kalman filter fall into two groups: time update equations and measurement update equations. The time update equations are responsible for projecting forward (in time) the current state and error covariance estimates to obtain the a priori estimates for the next time step. The measurement update equations are responsible for the feedback (i.e. for incorporating a new measurement into the a priori estimate to obtain an improved a posteriori estimate) [5]. The time update equations can also be considered as predictor equations, while the measurement update equations can also be considered as corrector equations.Therfore, the final estimation algorithm resembles a predictor-corrector algorithm for solving numerical problems. The specific equations for the time and measurement updates are presented below.

Time update equations

$$
\begin{aligned}
& \hat{\mathbf{x}}_{k}^{\prime}=\mathbf{A} \hat{\mathbf{x}}_{k-1}+\mathbf{B} \mathbf{u}_{k-1} \\
& \mathbf{P}_{k}^{\prime}=\mathbf{A} \mathbf{P}_{k-1} \mathbf{A}^{T}+\mathbf{Q}
\end{aligned}
$$

Again notice how the time update equations (2.50), (2.51) project the state and covariance estimates forward from time step $k-1$ to step $k$. A and $\mathbf{B}$ are from Eq. (2.38), while $\mathbf{Q}$ is from Eq. (2.40). Initial conditions for the filter are discussed in the earlier references.

Measurements update equations

$$
\mathbf{K}_{k}=\mathbf{P}^{\prime} \mathbf{H}\left(\mathbf{H P}^{\prime} \mathbf{H}^{T}+\mathbf{R}\right)^{-1}
$$




$$
\hat{\mathbf{x}}_{k}=\hat{\mathbf{x}}^{\prime}+\mathbf{K}_{k}\left(\mathbf{z}_{k}-\mathbf{H} \hat{\mathbf{x}}_{k}^{\prime}\right)
$$

$$
\mathbf{P}_{k}=\left(\mathbf{I}-\mathbf{K}_{k} \mathbf{H}\right) \mathbf{P}_{k}^{\prime}
$$

The first task during the measurement update is to compute the Kalman gain $\mathbf{K}_{k}$. Notice that the equation given here as Eq. (2.52) is the same as Eq. (2.47). The next step is actual to measure the process to obtain $\mathbf{z}_{k}$ and then to generate an a posteriori state estimated by incorporating the measurement as in Eq. (2.53). Again Eq. (2.53) is simply Eq. (2.44) repeated here for completeness. The final step is to obtain an a posteriori error covariance estimate via Eq. (2.54). After each time and measurement update pair, the process is repeated with the previous a posteriori estimates used to project or predict the new a priori estimates. This recursive nature is one of the very appealing features of the Kalman filter. It makes practical implementations much more feasible than, for example, an implementation of a Wiener filter (Brown 1983), which is designed to operate on all of the data directly for each estimate. The Kalman filter instead recursively conditions the current estimate on all of the past measurements. Fig. 2.11 offers a complete picture of the operation of the filter [5]. 


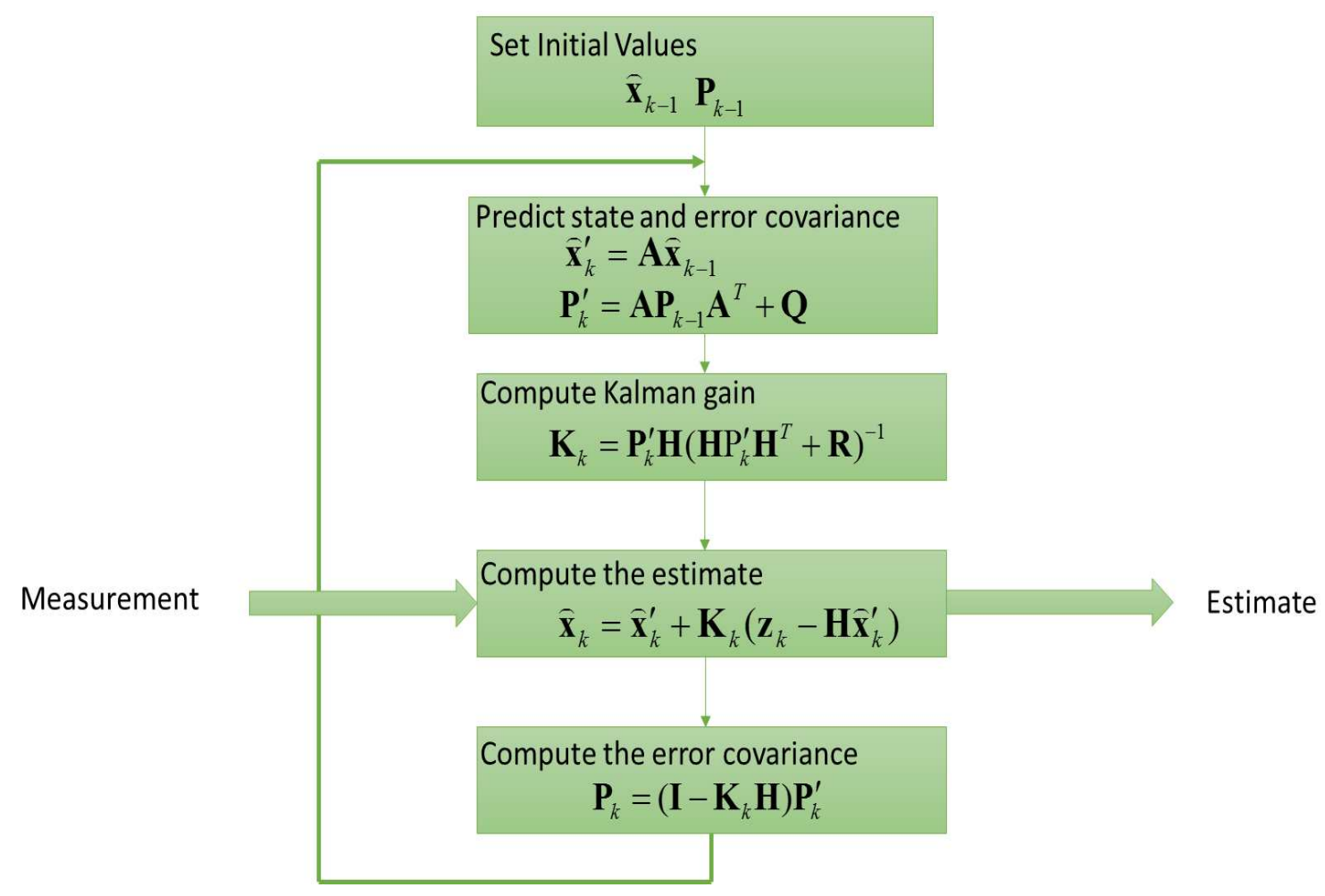

Figure 2.11: A Complete Picture of the Operation of Kalman Filter [5] 


\subsubsection{State Space Model of the MEMS Accelerometer Sensor Errors}

To estimate the sensor errors using Kalman filter, the sensor error model in subsubsection 2.1.1.2 has to be transformed to state space form. The errors of the sensor will be modeled as a 12-element state vector [16].

$$
[\mathbf{x}]=\left[\begin{array}{llllllllllll}
\theta_{x y} & \theta_{x z} & \theta_{y x} & \theta_{y z} & \theta_{z x} & \theta_{z y} & S F_{x} & S F_{y} & S F_{z} & \beta_{x} & \beta_{y} & \beta_{z}
\end{array}\right]^{T}
$$

where:

$\theta_{x y}=$ misalignment about $x$-axis reference to $y$-axis.

$\theta_{x z}=$ misalignment about $x$-axis reference to $z$-axis.

$\theta_{y x}=$ misalignment about $y$-axis reference to $x$-axis.

$\theta_{y z}=$ misalignment about $y$-axis reference to $z$-axis.

$\theta_{z x}=$ misalignment about $z$-axis reference to $x$-axis.

$\theta_{z y}=$ misalignment about $z$-axis reference to $y$-axis.

$S F_{x}=$ scale factor about $x$-axis.

$S F_{y}=$ scale factor about $y$-axis.

$S F_{z}=$ scale factor about $z$-axis.

$\beta_{x}=$ bias about $x$-axis.

$\beta_{y}=$ bias about $y$-axis.

$\beta_{z}=$ bias about $z$-axis.

On the other hand, linear measurement model can be modeled as:

$$
\mathbf{z}_{k}=\mathbf{H}_{k} \mathbf{x}_{k}+\mathbf{v}_{k}
$$


where:

$$
[\mathbf{H}]=\left[\begin{array}{cccccccccccc}
a_{y} & a_{z} & 0 & 0 & 0 & 0 & a_{x} & 0 & 0 & -1 & 0 & 0 \\
0 & 0 & a_{x} & a_{z} & 0 & 0 & 0 & a_{y} & 0 & 0 & -1 & 0 \\
0 & 0 & 0 & 0 & a_{x} & a_{y} & 0 & 0 & a_{z} & 0 & 0 & -1
\end{array}\right]
$$

where:

$$
\begin{aligned}
& a_{x}=\text { input acceleration about } x \text {-axis. } \\
& a_{y}=\text { input acceleration about } y \text {-axis. } \\
& a_{z}=\text { input acceleration about } z \text {-axis. }
\end{aligned}
$$

Kalam filter analysis procedure, as shown above, is used to determine the estimate $\mathbf{x}$ which represents the sensor errors. [20]. 


\section{Chapter 3}

\section{Test Equipment Design and Setup}

\subsection{Design Description}

The rotary motion is an important component of the testing system. A rotating turntable is used to provide the rotary motion. Moreover, a DC motor and a power supply are used to power and to drive the system. The testing system is powered by a power supply which its output is $30 \mathrm{~V}$ DC with a maximum current of $50 \mathrm{~A}$. Hence, the angular velocity of the turntable is directly dependent on the speed of the DC motor that is controlled by a speed controller. The general idea of the system is shown in Fig. 3.1.

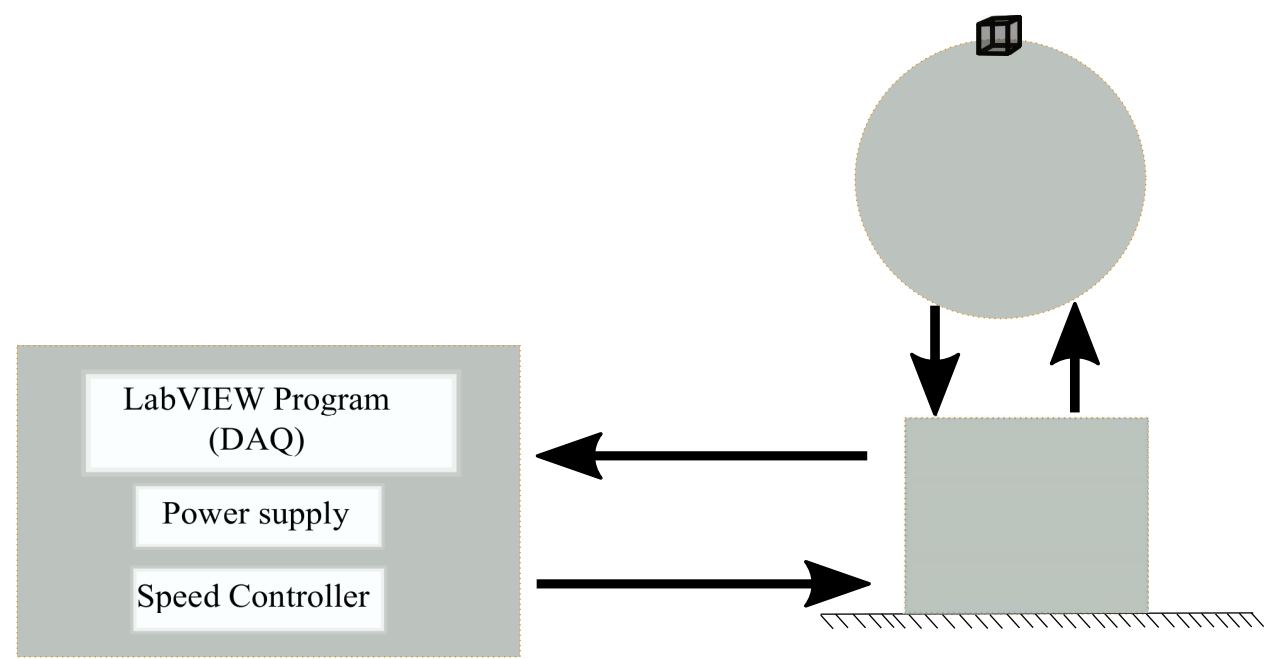

Figure 3.1: General Idea of the Test 


\subsubsection{Turntable}

This part is considered the largest part of the system because it is used to increase the range of the input acceleration. The diameter of this part is $90 \mathrm{~cm}$, and the thickness is $4 \mathrm{~cm}$ as shown in Fig. 3.2 .
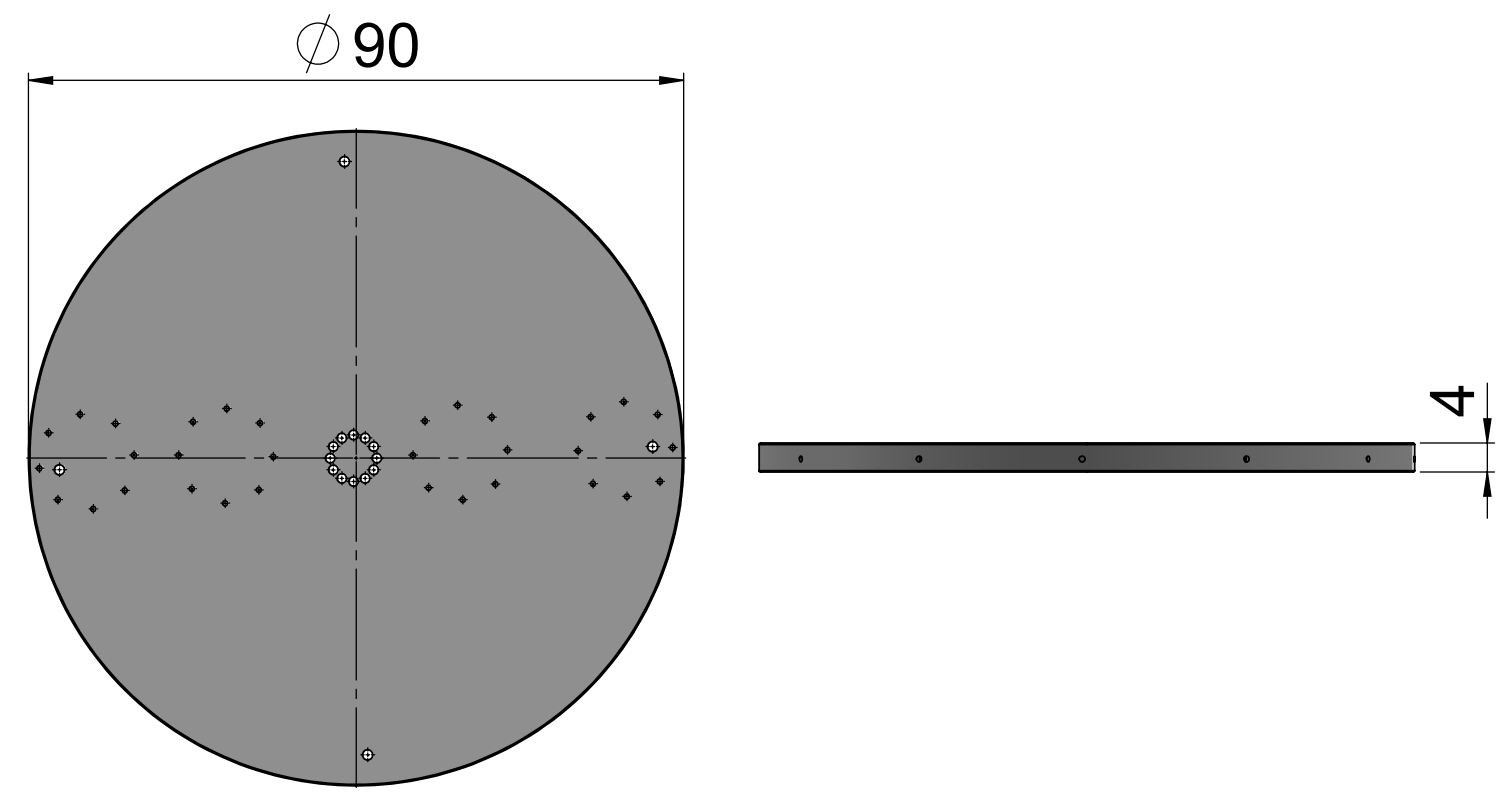

Figure 3.2: Dimensions of the Turntable

To increase the difference range of the input acceleration, the turntable has installation holes. These holes are used to control the value of radius which is responsible for the acceleration range. For example, if we want to increase or decrease the acceleration value, we need to adjust the radius, which is the distance from the accelerometer sensor to the center of the turntable as explained in Fig. 3.3. In order to get high accurate angular velocity, the speed controller should be used. Moreover, By adjusting the radius and the speed of the DC motor, the system would be able to generate a wide range of linear acceleration without gears. 

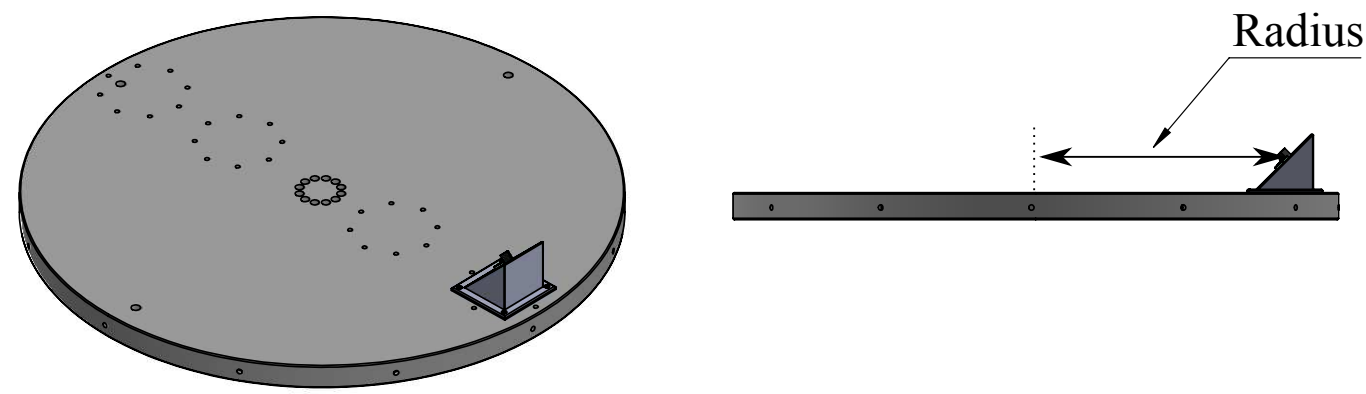

Figure 3.3: Limit Acceleration by Adjusting Radius

\subsubsection{Mounting the Sensor}

A mounting block was carefully fabricated within specified tolerances. Dual Axis Digital Angle Protractor with five magnetic sides was used to match the angles between the coordinates system of the sensor and the coordinates system of the testing system. High precise equipment was needed to mount the sensor with the testing system to avoid any misalignment sensitivity between the testing system and the sensor. Therefore, Dual Axis Digital Angle Protractor was used to get high accurate result for the testing system.

\subsubsection{Wiring the Turntable}

The electrical power, input, and output signals to the accelerometer sensor could be transferred through a slip ring. However, the output signals can also be transferred via wireless transmission. Since the analog signal could suffer some degradation by going through slip rings, the microprocessor could be implemented on the top of the table to collect data from the test, and then transform it to a digital signal through an A/D converter. These digital signals could be transferred to an acquisition computer 
to process them via slip rings or radio transmission. In this project, since the outputs of the sensor were digital signals, the slip rings were used without any interfere of noises and signal degradation.

\subsubsection{Motor Selection}

The high acceleration range of $\pm 50 \mathrm{~g}$ of the $3 \mathrm{D}$ axes test needs a high-performance DC motor. Moreover, due to the size and the weight of the turntable, the testing system had high moment of inertia. Table 3.1 illustrates the mean properties of the DC motor used in the current study. Therefore, a high torque DC motor was used. In order to avoid the signal instability of the decoder card that might be caused by the magnetic field of the electrical current, This DC motor was placed at a specific distance from the decoder card.

Table 3.1: Properties of the DC motor

\begin{tabular}{|c|c|}
\hline Voltage & $12 \mathrm{~V} \mathrm{DC}$ \\
\hline Speed & $1750 \mathrm{RPM}$ \\
\hline Power & $\frac{1}{2} \mathrm{H.P}$ \\
\hline Torque & 18 in.lbs \\
\hline $\begin{array}{c}\text { Full Load } \\
\text { Current }\end{array}$ & 39 Amps \\
\hline
\end{tabular}




\subsubsection{Accelerometer Sensor}

Analog device inertial sensor with the specifications listed in Table 4.2 was employed for the test [21].

Table 3.2: Analog inertial sensor (ADIS16400) specification

\begin{tabular}{|c|c|}
\hline \multicolumn{2}{|c|}{ ADIS16400 Accelerometer Sensor Specifications } \\
\hline Accelerometer Dynamic Range & $\pm 18 \mathrm{~g}$ \\
\hline $\begin{array}{c}\text { Accelerometer Initial } \\
\text { Sensitivity }\end{array}$ & $3.33( \pm 1 \%) \mathrm{mg}$ \\
\hline $\begin{array}{c}\text { Accelerometer Axis-to-Axis } \\
\text { Misalignment }\end{array}$ & \pm 0.0034 Radian \\
\hline $\begin{array}{c}\text { Accelerometer Axis-to-frame } \\
\text { (package) }\end{array}$ & \pm 0.0087 Radian \\
\hline Accelerometer Nonlinearity & $0.1 \%$ of FS \\
\hline
\end{tabular}




\subsection{Velocity Measurement Device Selection}

Analog and digital devices are commonly used for motion measurement. The analog devices generate the voltage or current output which is proportional to the velocity of the motor. However, The values of the analog signal are not reliable at low-speed motion because of the noise generated. Therefore, The analog devices are not accurate in measuring the slow velocity. On the other hand, the motion creates a stream of pulses equivalent to the magnitude and direction of the motion. The digital sensor are used to measure this stream of pulses. The most important factor for getting accurate measurement is the resolution of the sensors. The position rotation can be measured by using a rotary encoder that is one of the digital devices. There are many ways to determine the angular velocity. One of these ways is by measuring the difference in the position of rotation over the time between two samples. in order to have velocity that is closed to the continuous form, the resolution of the measured velocity has to be increased as much as necessary. The rotary encoder consists of a rotary disk that is attached to a rotational part and a reading head. The encoder signal is generated when the marking lines on the encoder disk move to block and unblock the light to the receiver creating an interference pattern. The resolution of the system is defined as the number of lines per revolution. The encoder is divided into two types; absolute and incremental encoder. As shown in Fig. 3.4, an absolute encoder can provide the absolute angular position in one rotation, but the incremental encoder gives the change in position. Moreover, the incremental encoder provides finer resolution. Although one output pulse stream is useful for measuring speed, it does not indicate the direction of rotation (i.e., clockwise vs. counter-clockwise) of an incremental encoder. To get around this problem, an incremental encoder has two disks of alternating regions, each with its own photo emitter and detector, that allow 
two pulse streams to be produced simultaneously. The two pulse streams ("clocks") can be used to determine both velocity and direction. This is made possible by an intentional misalignment of the two disks that causes a 90 degree phase difference between their clocks. An incremental encoder that produces two clocks (typically designated A and B) having a 90 degree phase difference is said to be "quadrature" encoded. In the case of a quadrature incremental encoder, the A clock phase will lead $\mathrm{B}$ for one direction of rotation, and $\mathrm{B}$ will lead $\mathrm{A}$ when the encoder rotates in the opposite direction. A quadrature encoder's rotational direction can be determined by detecting the phase relationship between the A and B clocks, while its speed can be detected by measuring the frequency of either clock.

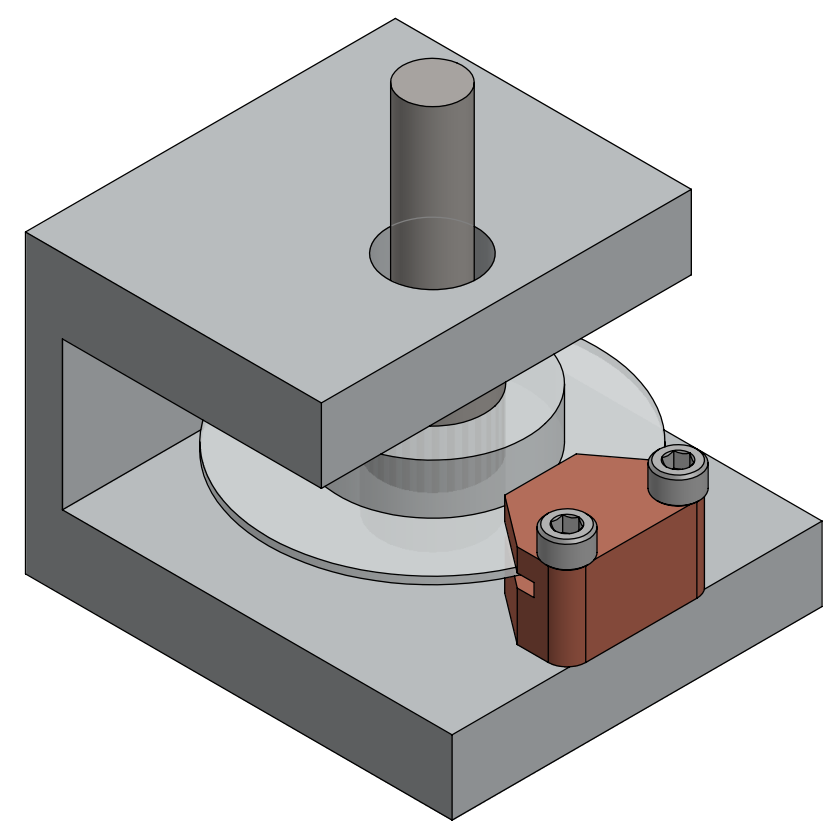

Figure 3.4: Virtual Absolute Encoder

The widely used techniques for measuring the rotational velocity with the optical encoder are by counting the number of pulses per sampling time, or by measuring 
pulse period that is defined as the time between two consecutive pulses. The second technique more suitable for the high velocity measurement [22]. However, the first technique (M method) is the most widely used method to measure the angular velocity from encoder pulses. Moreover, M method was used with this project because of the accuracy of this technique is governed by the resolution of the encoder [17]. The principle of velocity measurement is depicted in Fig. 3.5 [6].

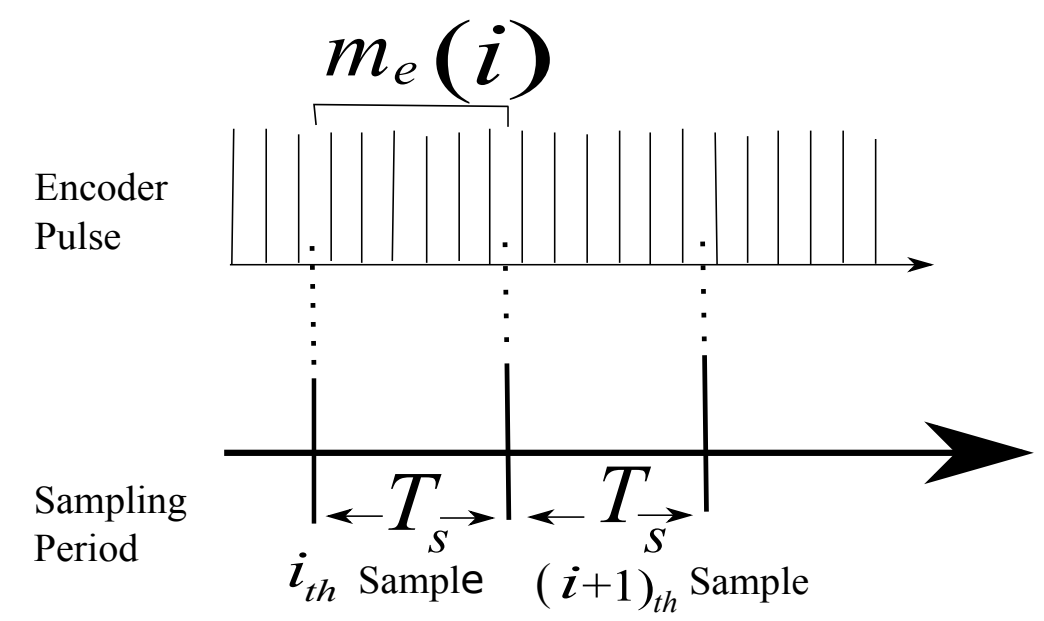

Figure 3.5: Principle of Velocity Measurement Method [6]

The angular velocity is calculated by finite-difference method using the constant sampling time and the number of pulses per sampling time.

$$
\begin{gathered}
\bar{\omega}=\frac{2 \pi m_{e}}{P T_{s}} \\
Q_{v}=\frac{2 \pi}{P T_{s}} \\
T_{m}=T_{s}
\end{gathered}
$$


where:

$\omega=$ angular velocity.

$m_{e}=$ the number of pulses per sampling time.

$P=$ the number of counts per revolution.

$Q_{v}=$ the absolute velocity resolution.

$T_{s}=$ the time interval used to count a pulse number periodically (sampling time).

$T_{m}=$ the measurement time used to calculate velocity based on pulse count within one or more sampling time. 


\subsection{Kinematic Model}

Three angles are required to specify the orientation of a rigid body relative to an inertial frame. The choice is not unique, but there are two sets in commonly used: the Euler angles and the yaw, pitch and roll angles. The three Euler angles give the orientation of a rigid, orthogonal $x, y$ and $z$ frame of reference relative to the 1, 2 and 3 inertial frame of reference. The orthogonal triad of unit vectors parallel to the inertial axes 1,2 and 3 are $\hat{\mathbf{I}}, \hat{\mathbf{J}}$ and $\hat{\mathbf{K}}$ respectively. The orthogonal triad of unit vectors lay along the axes of the 1,2 and 3 frame are $\hat{\mathbf{i}}, \hat{\mathbf{j}}$ and $\hat{\mathbf{k}}$ respectively. Fig. 3.6 shows the $\hat{\mathbf{I}}, \hat{\mathbf{J}}$ and $\hat{\mathbf{K}}$ triad and the $\hat{\mathbf{i}}, \hat{\mathbf{j}}$ and $\hat{\mathbf{k}}$ triad, along with the three successive rotations required to bring unit vectors initially aligned with $\hat{\mathbf{I}}, \hat{\mathbf{J}}$ and $\hat{\mathbf{K}}$ into alignment with $\hat{\mathbf{i}}, \hat{\mathbf{j}}$ and $\hat{\mathbf{k}}[7]$. The $x y$ plane intersects the 12 plane along a line defined by the unit vector $\hat{\mathbf{i}}$. The first rotation (1) is around the $\hat{\mathbf{K}}$ axis through the Euler angle. $\phi$ rotates the $\hat{\mathbf{I}}, \hat{\mathbf{J}}$ directions into the $\hat{\mathbf{i}}, \hat{\mathbf{j}}$ directions. As shown in Fig. 3.6. 


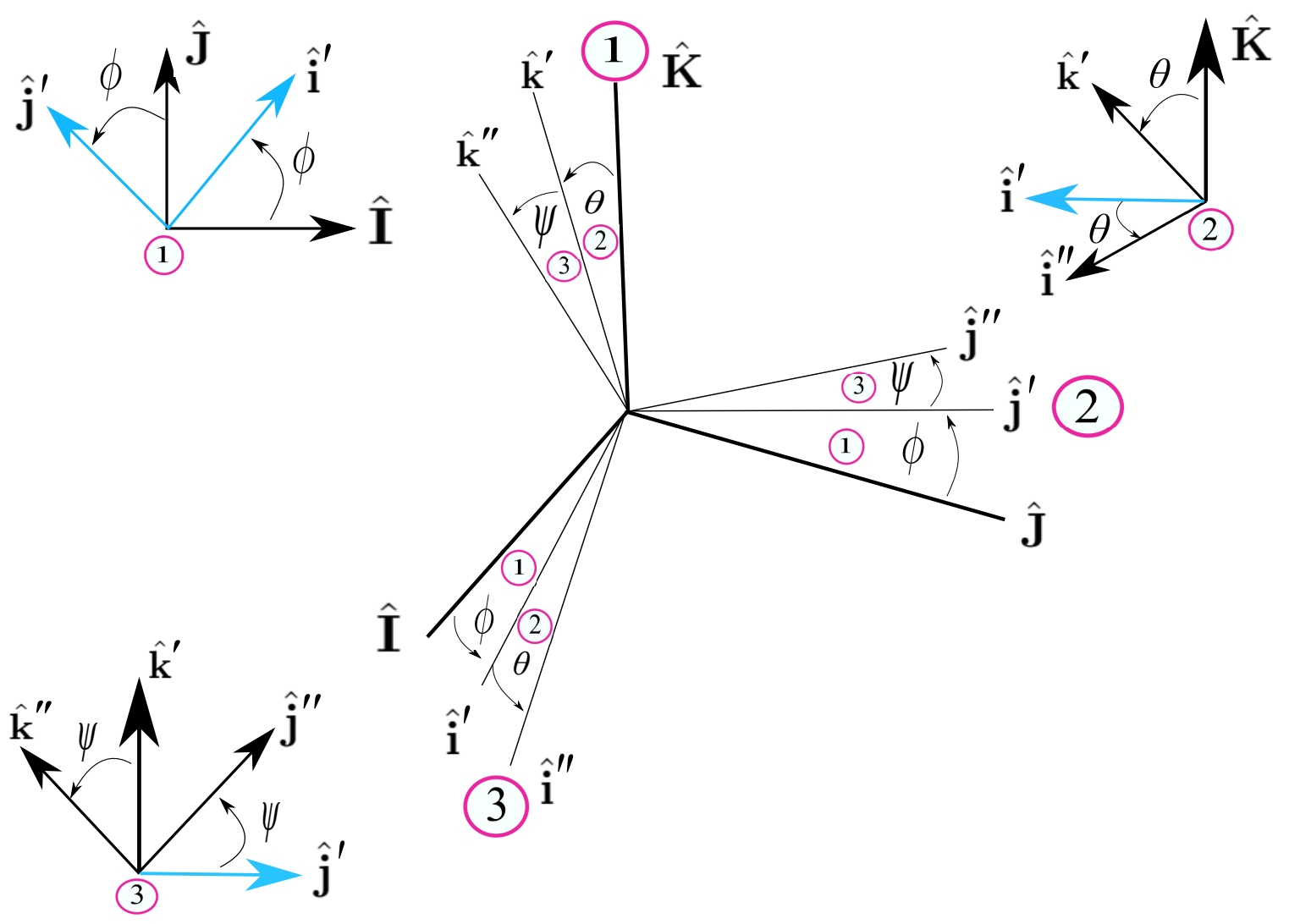

Figure 3.6: The Euler Angles [7]

where:

$$
\begin{aligned}
& (\prime)=\text { first rotation from axis. } \\
& (\prime \prime)=\text { second rotation from axis. }
\end{aligned}
$$

There are three rotations around three axes. Therefore, the first rotation about 3-axis can be expressed by:

$$
\mathbf{R}_{3}=\text { Rotation about } 3 \text {-axis with }(\phi) \text { angle }
$$




$$
\mathbf{R}_{3}(\phi)=\left[\begin{array}{ccc}
\cos (\phi) & \sin (\phi) & 0 \\
-\sin (\phi) & \cos (\phi) & 0 \\
0 & 0 & 1
\end{array}\right]
$$

The second rotation about 2-axis can be expressed by;

$\mathbf{R}_{2}=$ Rotation about 2-axis with $(\theta)$ angle

$$
\mathbf{R}_{2}(\theta)=\left[\begin{array}{ccc}
\cos (\theta) & 0 & -\sin (\theta) \\
0 & 1 & 0 \\
\sin (\theta) & 0 & \cos (\theta)
\end{array}\right]
$$

And the third rotation about 1-axis can be expressed by;

$\mathbf{R}_{1}=$ Rotation about 1-axis with $(\psi)$ angle

$$
\mathbf{R}_{1}(\psi)=\left[\begin{array}{ccc}
1 & 0 & 0 \\
0 & \cos (\psi) & \sin (\psi) \\
0 & -\sin (\psi) & \cos (\psi)
\end{array}\right]
$$

Finally, it is worth noting that the transformation matrix $[\mathrm{Q}] X x$ from the inertial 1, 2 and 3 frame into the moving $x, y$ and $z$ frame is the product of the three rotation matrices given by Eq. (3.4), (3.5) and (3.6). Thus, 


\section{$[\mathbf{Q}] X x=[\mathbf{R} 3(\phi)][\mathbf{R} 1(\psi)][\mathbf{R} 2(\theta)]$}

$$
[\mathbf{T}]=\left[\begin{array}{ccc}
\sin (\psi) \sin (\phi) \sin (\theta)+\cos (\theta) \cos (\phi), & \cos (\psi) \sin (\phi), & \sin (\psi) \cos (\theta) \sin (\phi)-\sin (\theta) \cos (\phi) \\
\sin (\psi) \sin (\theta) \cos (\phi)-\cos (\theta) \sin (\phi), & \cos (\psi) \cos (\phi), & \sin (\psi) \cos (\theta) \cos (\phi)+\sin (\theta) \sin (\phi) \\
\cos (\psi) \sin (\theta), & -\sin (\psi), & \cos (\psi) \cos (\theta)
\end{array}\right]
$$




\subsection{The Testing Methodology of Simulation}

Kalman filter was used to determine sensor errors. The circular motion of the system provides the input rate to generate the acceleration inputs in three sensitive axes of the sensor simultaneously. National Instruments USB-4851 I2C/SPI interface was used to collect measurement data of the accelerometer sensor and reference input angular velocity. Gurley 7700A02048R12U0175N encoder with the base resolution of 2048-line disc and its 11 bits upscale decoder VJ11S08U0CN has the total resolution range of 19 bits (524288 steps / rev) which can be able to measure a $0.0007 \mathrm{deg} / \mathrm{step}$ was used to measure the reference angular velocity inputs [23]. To generate the input acceleration vary from $2.32 \times 10^{-9}$ to $50 \mathrm{~g}$ on three axes of the sensor. 


\subsection{Verification Study}

Prior to the testing process, it was important to examine some aspects of the testing system in order to ensure that any errors taken place with these aspects would not significantly affect the test results. The verification study was conducted in terms the Kalman filter and the static balance of the shaft and the turntable.

\subsubsection{Kalman Filter Parameters}

To estimate the parameters for the kalman filter, the system model is used. First, it is needed to estimate the initial state for the system. Since the system is started from zero, the initial state will be zeros. The matrix $\mathbf{A}$ is the behavior of the system with time. It depends on the sampling time of the system as explained in Eq. (2.38). In Eq. (2.40) and Eq. (2.41), $\mathbf{Q}$ and $\mathbf{R}$ values can be obtained from the manual sheet of the sensors [21]. These values depend on the noise density and bandwidth for the sensors. Moreover, the matrix $\mathbf{H}$ in the Kalman filter is the measurement matrix that can be estimated from the relationship between measured values from the sensor and the predict values as showed in Eq. (2.39).

\subsubsection{Static Testing - Balance}

There are two types of static balance for the system:

\section{A) Using Dail Gage and Dual-Axis Digital Protector}

As shown in Fig. 3.7, the static balancing process was conducted for both the top and the side of the shaft by using a dial gage. For the turntable, a dial gage and a dual-axis digital protector were used to perform the static balance testing. The testing results for the shaft with a dial gage showed that the error of the shaft was 
0.0015 inch and the error of the turntable was 0.0035 inch. These values of the errors were measured by mounting the dial gage at the static reference point outside of the testing system and the turntable or the shaft was rotated to measure the maximum value of the imbalance.

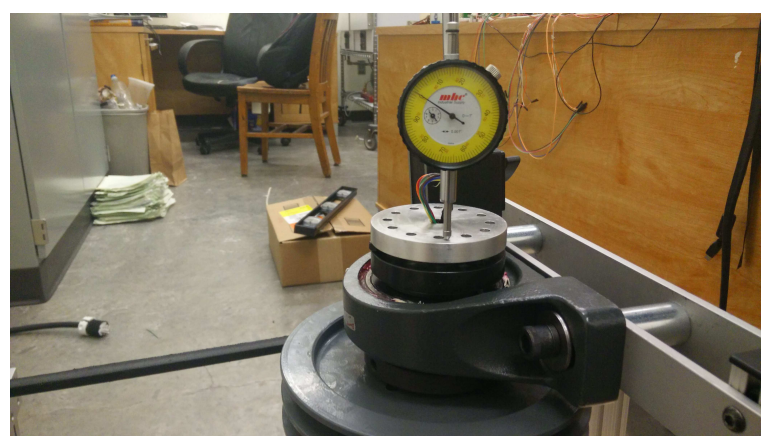

Figure 3.7: Shaft Balance

\section{B) Verify the Balance of the Turntable with Accelerometer Sensor}

As shown in Fig. 3.8, the misalignment of the accelerometer sensor was used to check the balance of the turntable. The accelerometer sensor was placed at several points on the turntable. The results of the misalignment of the sensor about zx and zy were used to estimate the imbalance of the turntable. To conduct the misalignment testing, the turntable was divided by two imaginary lines as shown in Fig. 3.9. One of the two lines was drawn so that it passed through the actual location of the sensor from the real test. Several of testing points were selected along those two lines. Each testing points represents the location at witch the misalignment of the turntable was measured by using the accelerometer sensor. The static testing points were labeled as $1,2,3, \ldots ., 8$ as shown in Fig. 3.9. The values of the misalignment angle $(\lambda)$ of the turntable at points 4 and 5 are $-0.12^{\circ}$ and $-0.09^{\circ}$, respectively. They were calculated as the difference between the maximum misalignment on the line and 
the misalignment of the sensor with ideal axis. The maximum misalignment of the line on the turntable was calculated by the Least of Square fit between the input acceleration on the z-axis due to gravity and the output acceleration on the $x$-axis and $y$-axis without rotation (static). The negative signs indicate that inclination of the turntable at the specified points is downward.

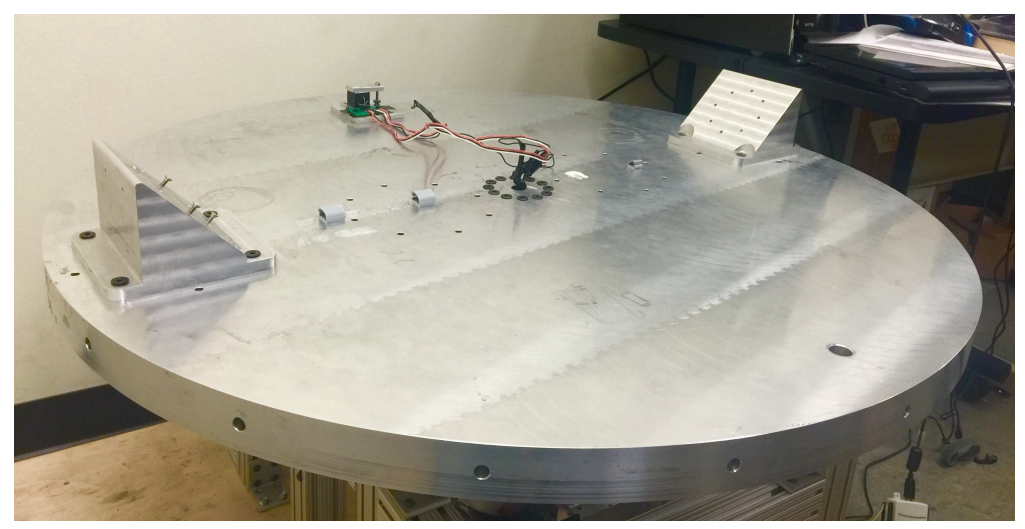

Figure 3.8: Balance of the Turntable with Accelerometer Sensor

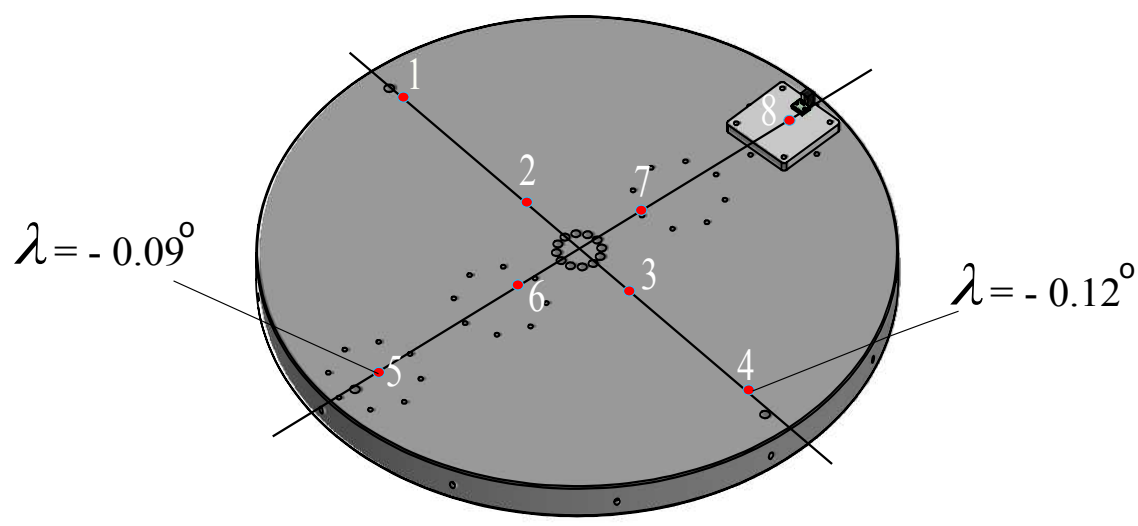

Figure 3.9: Static Balance Testing of the Turntable 


\section{Chapter 4}

\section{Results of Error Analysis of MEMS Accelerometer Sensor and Testing}

Although the microfabrication is very accurate, there is no way to make a perfect sensor that has no bias or affect other errors. The sensing for the multi-axes MEMS accelerometer sensor, bias offset, scale factor error, axis misalignment are three main errors that mostly contribute to error in sensor measurement. Bias offset is the output of the sensor when the input is zero as shown in Fig. 4.1. The scale factor is the ratio of the change in the input intended to be measured. The scale factor was normalized to 1. Aging or manufacturing tolerance could cause scale factor to be around 1. Axis misalignment is an assembly error such that the sensing axis of the accelerometer sensor is misaligned with respect to the reference axis. 


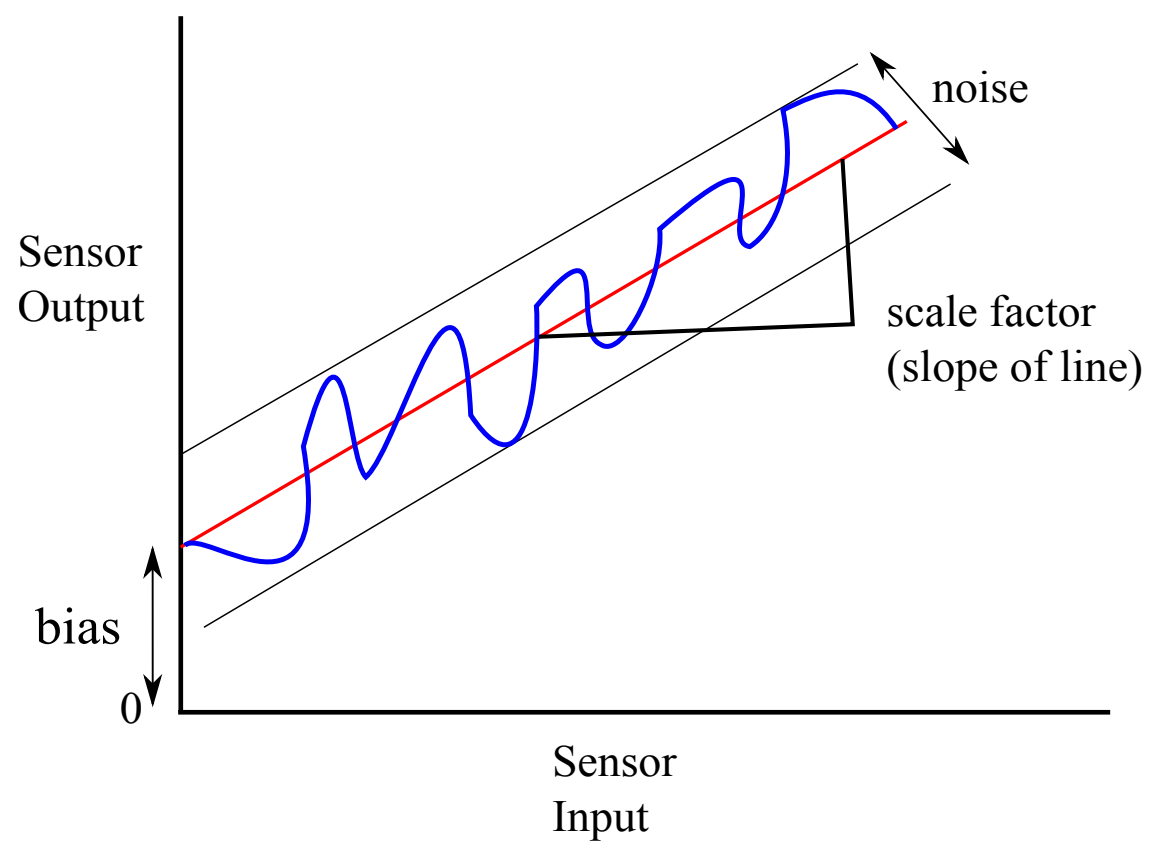

Figure 4.1: Common Errors in Accelerometer Sensor [8] 


\subsection{Three-dimensional Testing System.}

The 3-D Accelerometer testing system was designed as showed in Fig. 4.2.

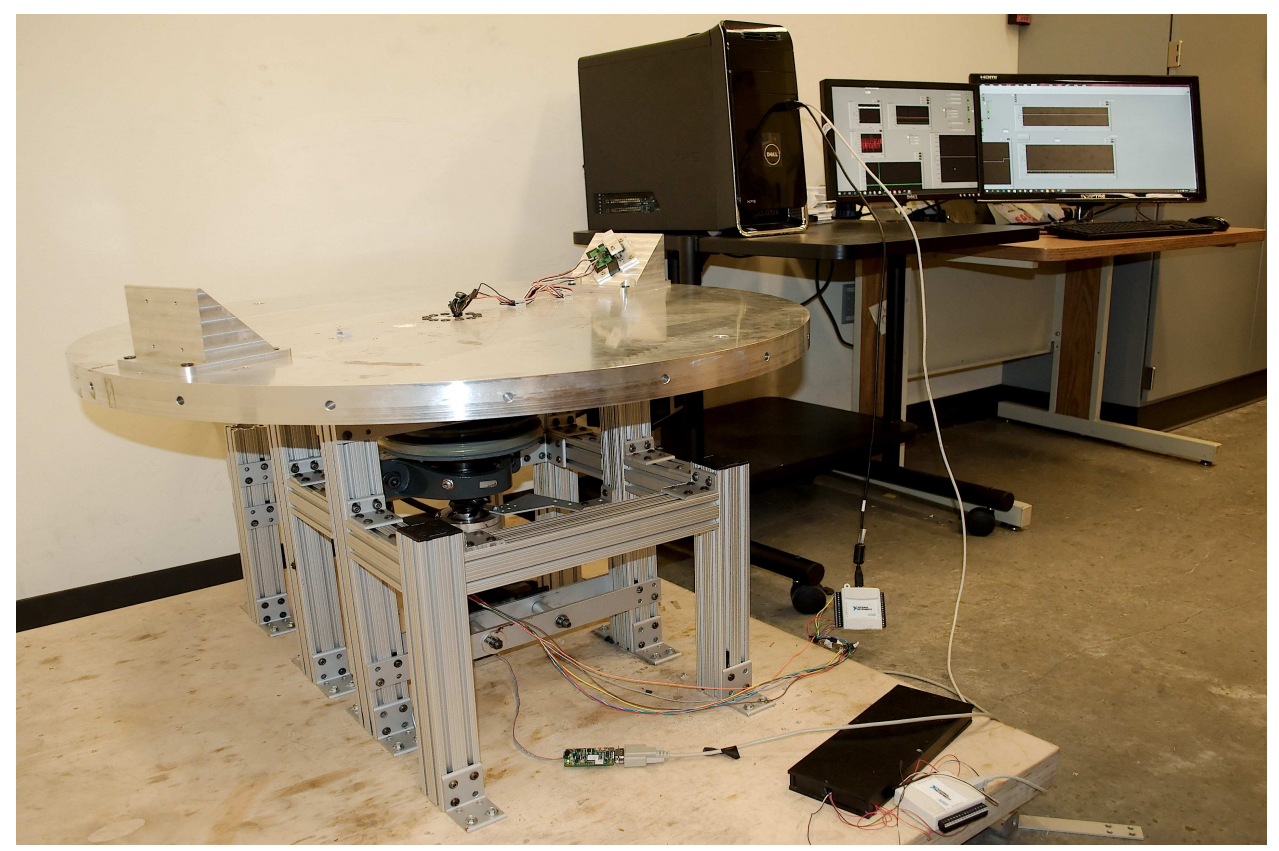

Figure 4.2: $3 \mathrm{D}$ Testing Machine

This testing machine was designed mainly for single/multi-axis MEMS accelerometer test. This testing machine provides precise continuous uniform angular rate motion about a spine axis. All operations and controls were computerized based on Labview ${ }^{M T}$. The capability of this 3-D MEMS accelerometer testing system is given in Table 4.1. 
Table 4.1: Acceleration testing machine properties

\begin{tabular}{|c|c|}
\hline $\begin{array}{c}\text { Acceleration Rate about All } \\
\text { Axes }\end{array}$ & $\begin{array}{c}\text { Min: } 2.32 \times 10^{-9} \\
\text { Max: } 50 \mathrm{~g}\end{array}$ \\
\hline \multirow{3}{*}{ Angular Position Measurement } & $\begin{array}{c}19 \text { bits resolution Absolute } \\
\text { encoder } 524288 \text { lines/rev } \\
(2.5 \operatorname{arcsec} / \text { line })\end{array}$ \\
\hline Position Accuracy & \pm 5.2 arcsec \\
\hline Slip-ring & 24 wires \\
\hline Control Software & Based on Labview ${ }^{M T}$ \\
\hline
\end{tabular}




\subsection{Data Processing}

The data was collected by using a program developed in Labview ${ }^{M T}$ and saved on a text file. The collected raw data was processed by using Matlab program. The program collects information about the turntable such as position in counts, position in degrees and angular velocity in degrees per second of the turntable. Along with that, acceleration measurements of the sensor along $x, y$, and $z$-axis were collected. During any testing mode, the turntable is rotated in both directions; clockwise and counterclockwise. The turntable is rotated in each direction at different magnitudes of angular velocity. Depending on the positioning of the accelerometer, this exposes the sensor to one or any different magnitudes of acceleration. 


\subsection{3-D Testing Results \& Discussion}

\subsubsection{Measured and Output}

\subsubsection{Measured vs. Output Acceleration along x, y and z Axes.}

Fig. 4.5 shows the change in the acceleration from encoder and output acceleration from sensor with time for $x$-axis. It can be seen from this figure that the measured acceleration from the encoder matches the sensor acceleration output. Similarly, this observation applies for the case of $y$-axis and $z$-axis as shown in Fig. 4.6 and Fig. 4.7 respectively.

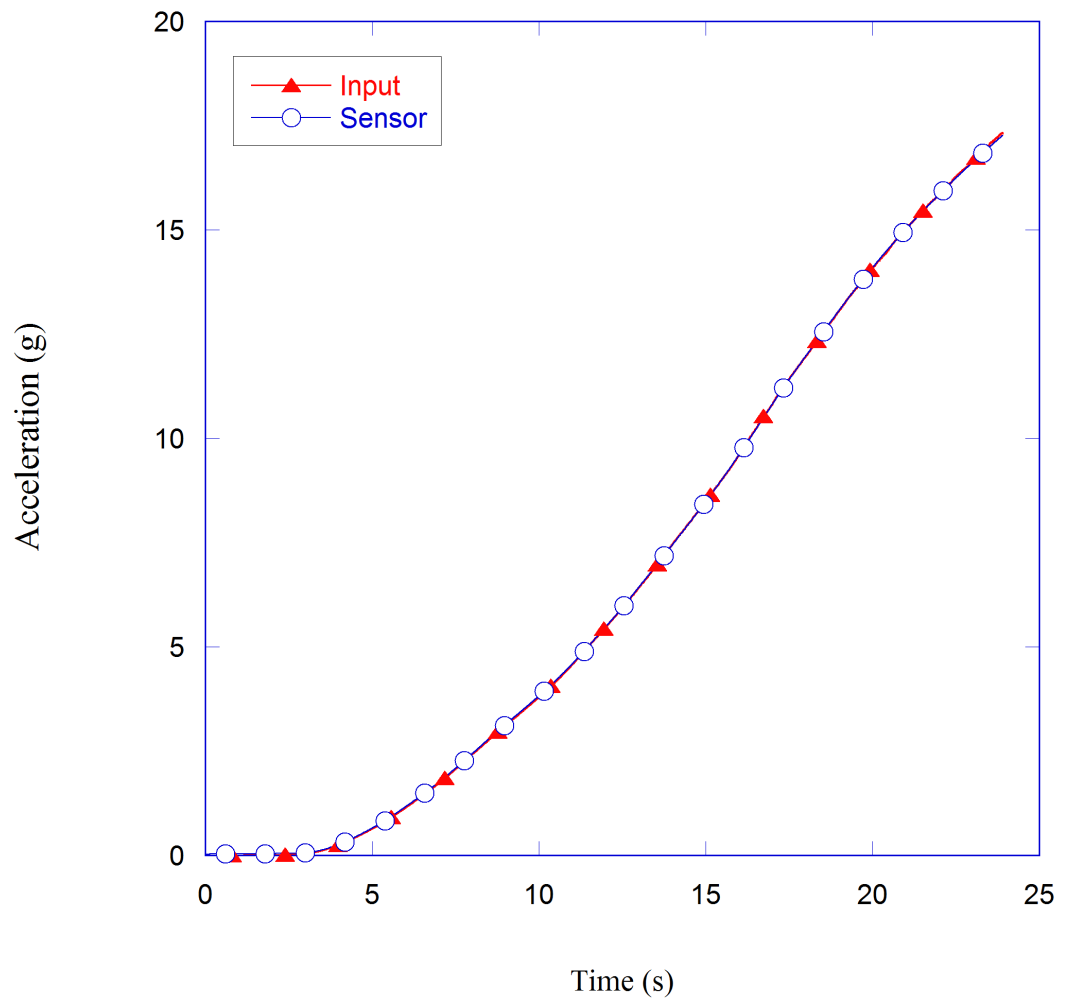

Figure 4.3: Acceleration Input and Sensor Output about $x$-Axis vs. Time 


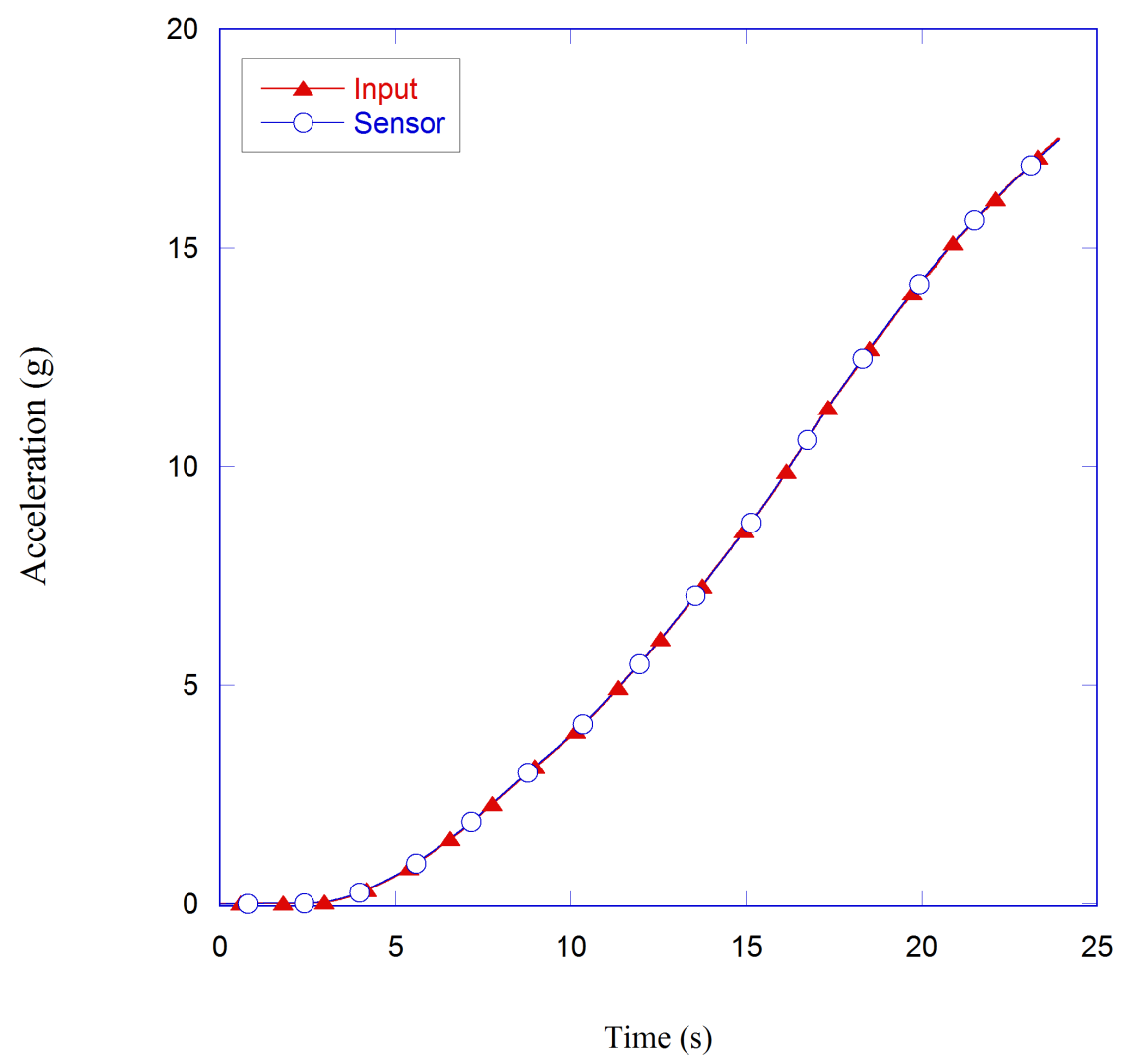

Figure 4.4: Acceleration Input and Sensor Output about $y$-Axis vs. Time 


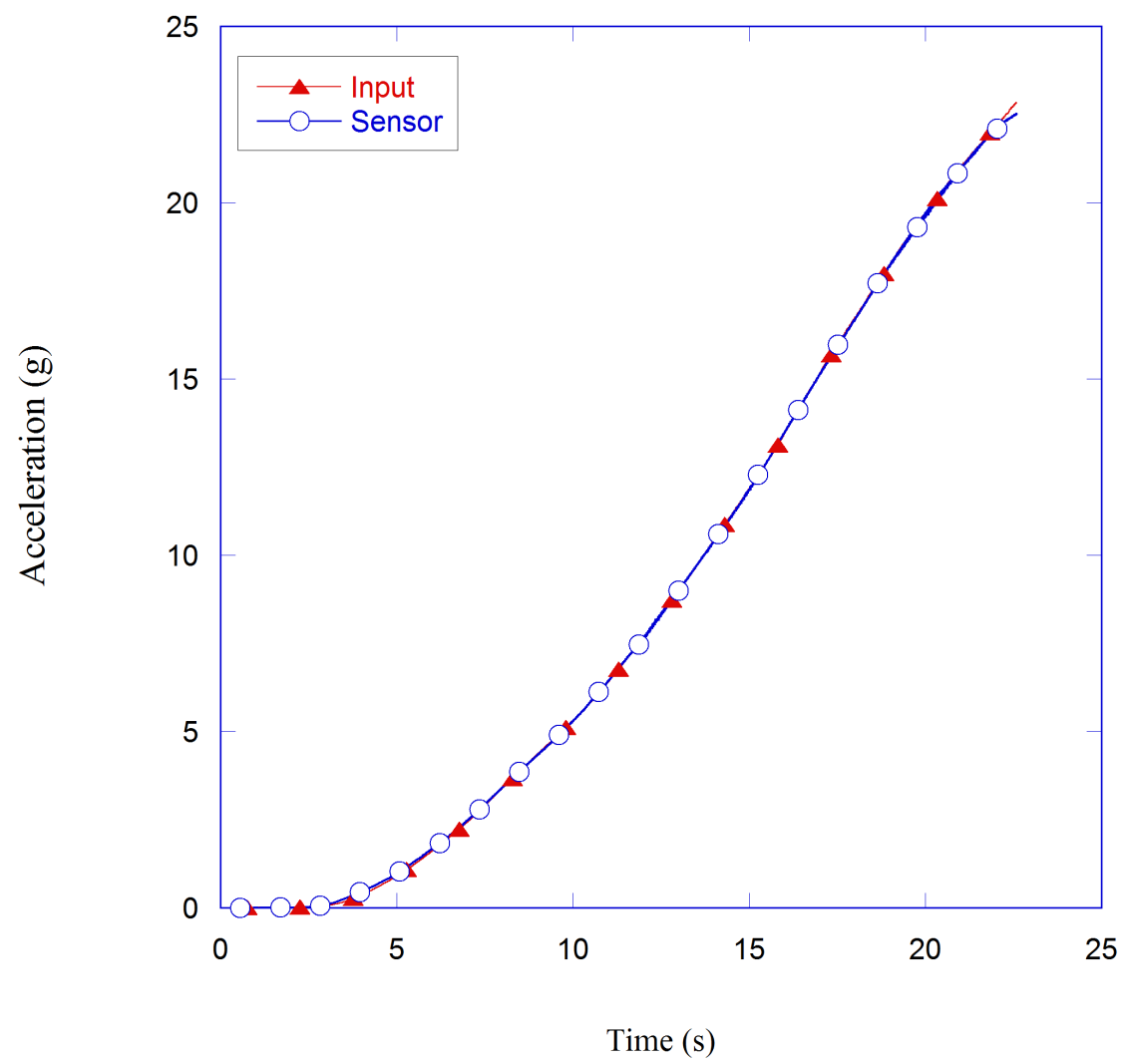

Figure 4.5: Acceleration Input and Sensor Output about $z$-Axis vs. Time 


\subsubsection{Measured Acceleration around $\mathrm{x}, \mathrm{y}$ and $\mathrm{z}$ Axes with the Kalman Filter}

Fig. 4.8 shows the change in the acceleration from encoder with time for $x$-axis after processing the data with Kalman filter. It can be seen from this figure that using the Kalman filter eliminated the noise in the raw data resulting in more accurate values of aceeleration and closer to the real values of the encoder. The same process was followed with raw data of the accelerometer sensor as shown in Fig. 4.9. The condition of the test was 23 second testing time and 304 RPM angular velocity in the room temperature. For better illustration of using the Kalman filter, a close-up view of a segment of the output acceleration along the $x$-Axis is shown in Fig. 4.10. From this figure, it can be clearly noted that the accuracy of the input and output data were improved by using the Kalman Filter and the noise was removed.
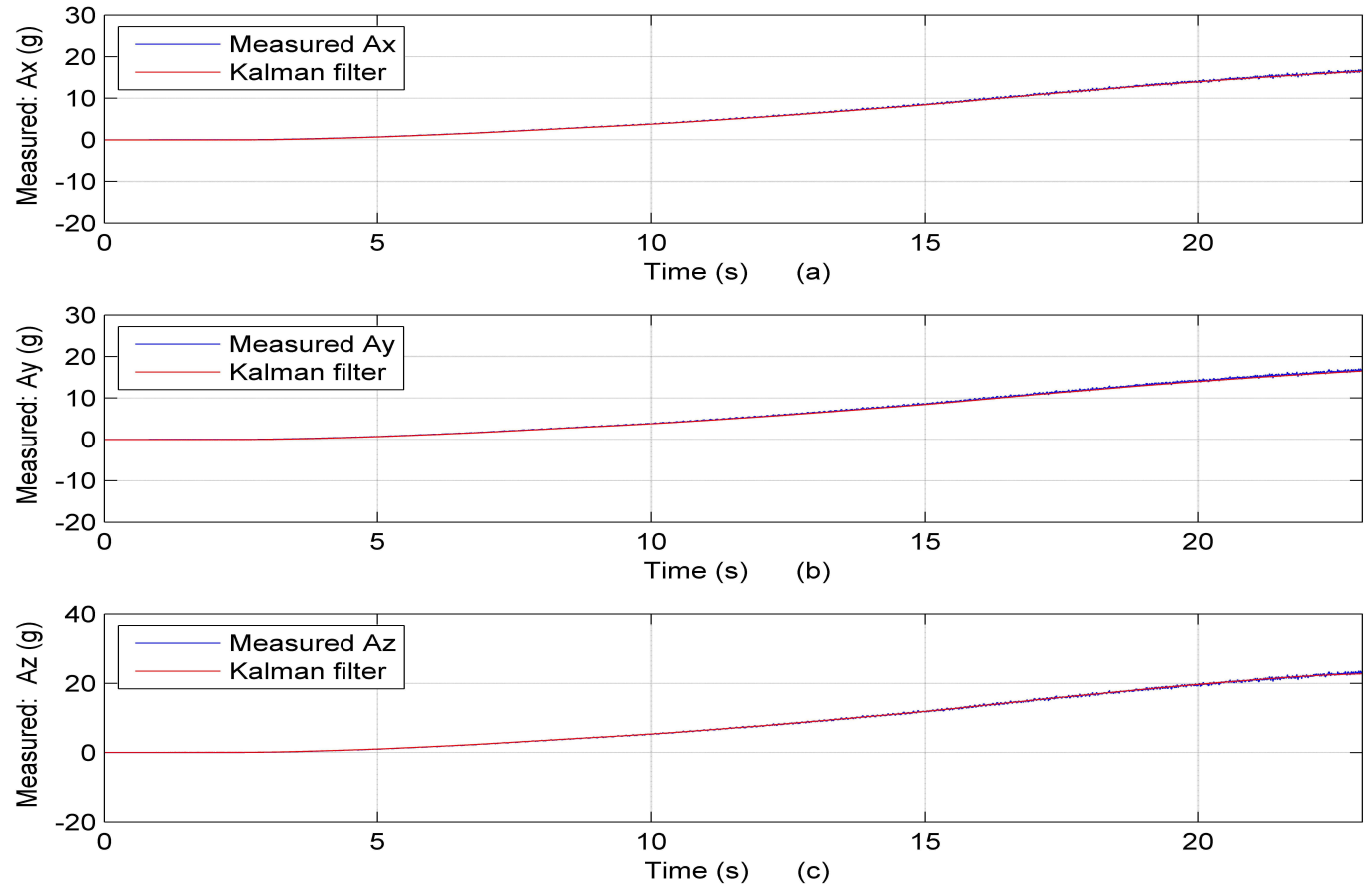

Figure 4.6: Measured Acceleration around $x, y$ and $z$ Axes with the Kalman Filter 


\subsubsection{Sensor Outputs Along $\mathrm{x}, \mathrm{y}$ and $\mathrm{z}$ Axes with the Kalman Filter}
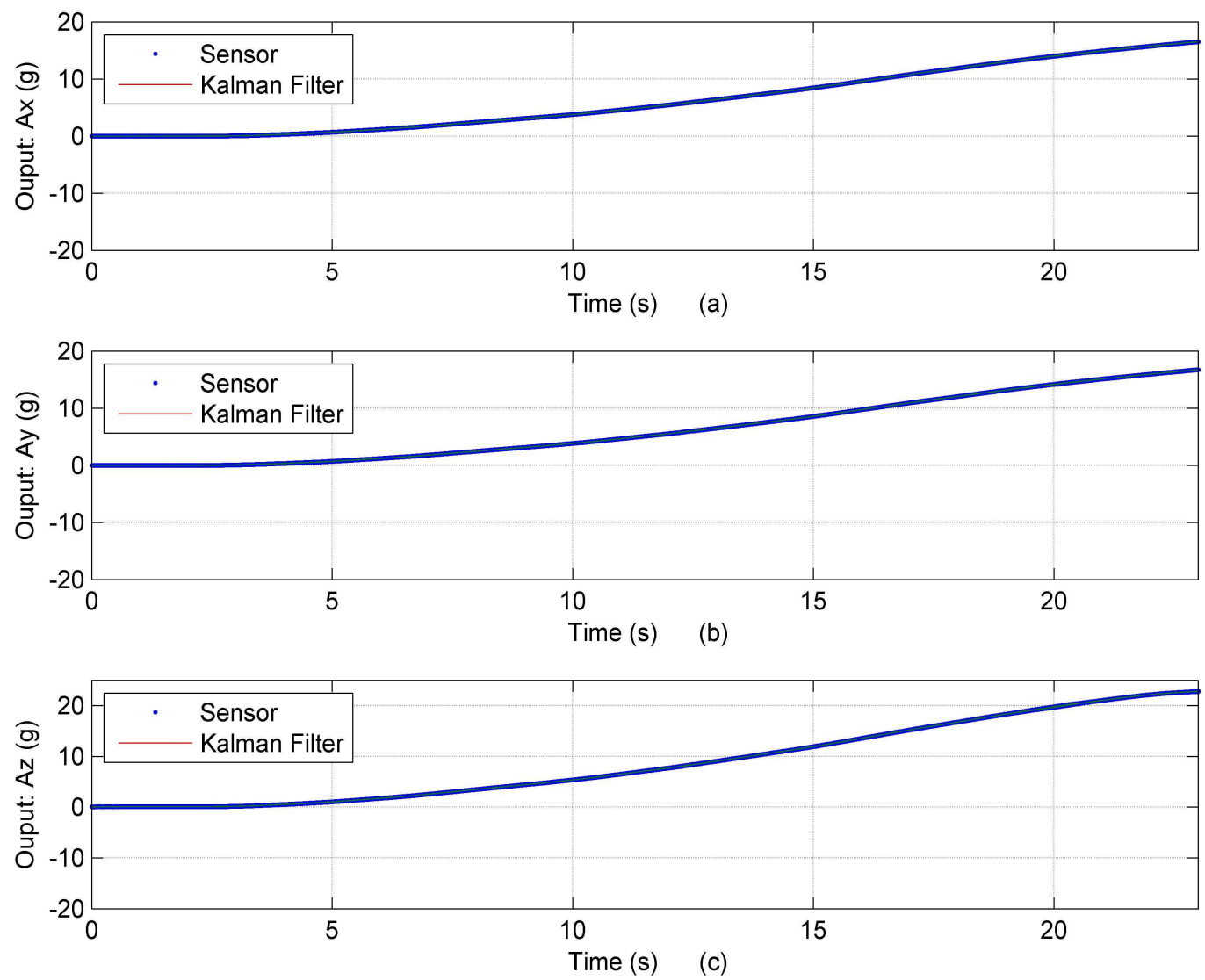

Figure 4.7: Sensor Outputs Along $x, y$ and $z$ Axes with the Kalman Filter 


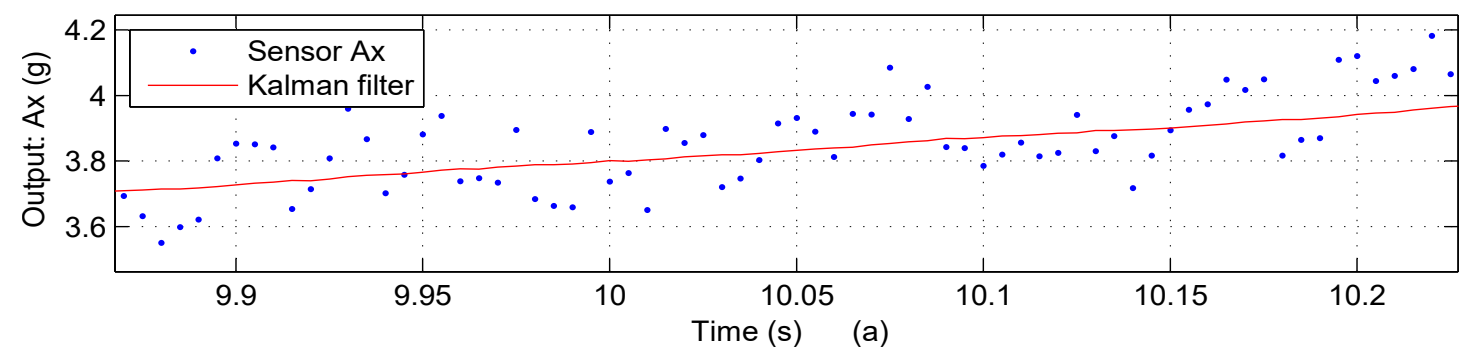

Figure 4.8: Output Acceleration around $x$-Axis with Raw Data and Kalman Filter

\subsubsection{Estimation of Errors}

\subsubsection{Sensor Bias Estimation Along $\mathrm{x}, \mathrm{y}$, and $\mathrm{z}$ Axes}

Fig. 4.11 shows the bias error around $x$-axis, $y$-axis, and $z$-axis. The bias error is the difference between the real value and the output. As shown in plot (a) in Fig. 4.11 , the bias error around $x$ is $0.0384 \mathrm{~g}$. Some values of the error up the tolerance of the sensor because the system is not calibrated yet. It can be seen from this figure that there are some instability in the first two second. Moreover, the error is changed at the first two second to be about $3 \%$ from the real error for the some axes. This value of the error was due to the Kalman gain. The Kalman gain needs more data points to be stable in order to find the exact error. This instability is related to initial estimations of the errors in the Kalman filter, and the instability of the error needs 2-3 seconds in order to reach the steady state. 

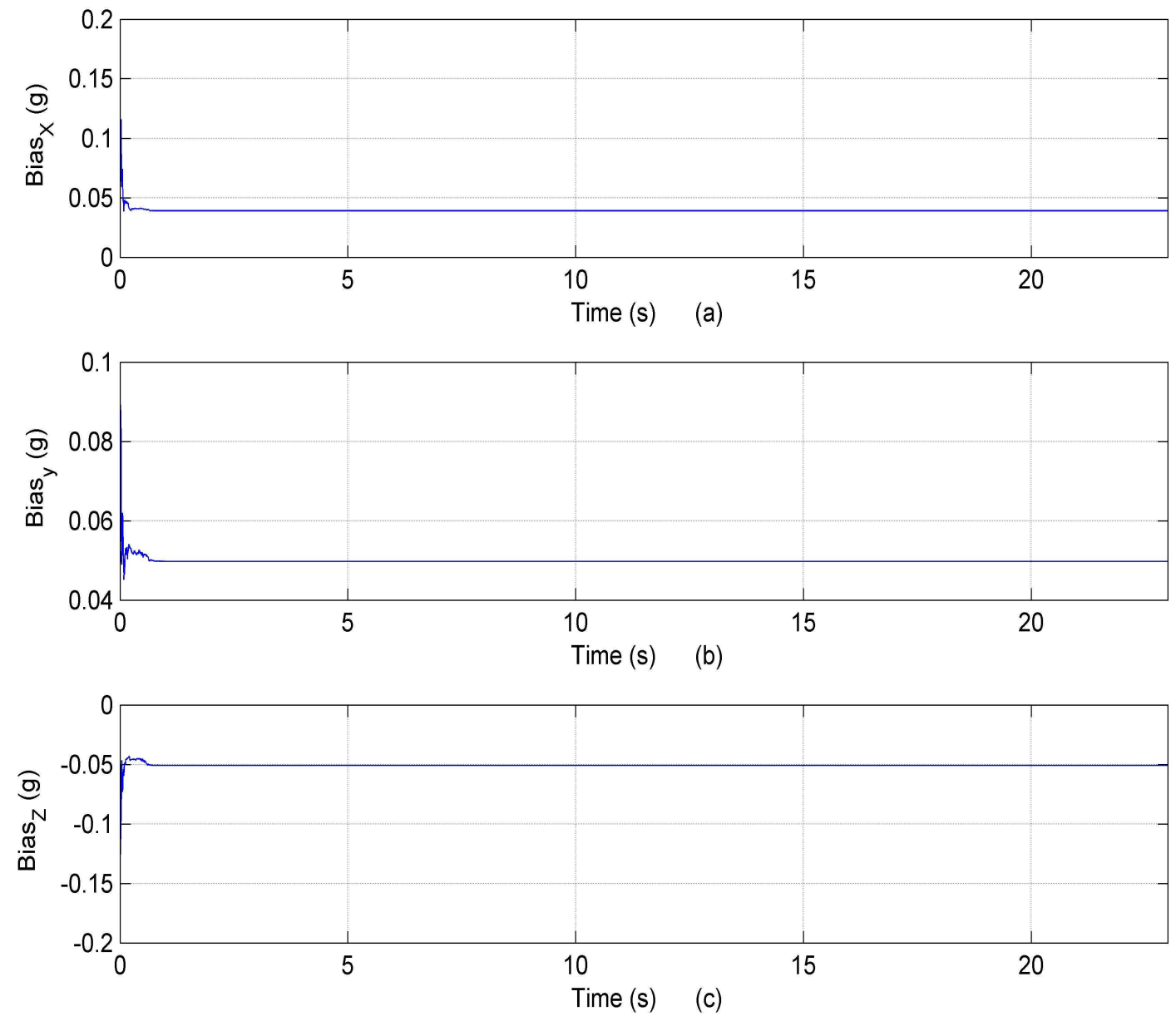

Figure 4.9: Sensor Bias Estimation Along $x, y$, and $z$ Axes 


\subsubsection{Scale Factor Estimation Along $\mathrm{x}, \mathrm{y}$, and $\mathrm{z}$ Axes}

Fig. 4.12 shows the Scale Factor error around three axes; $x$-axis, $y$-axis, and $z$-axis. The Scale factor error is the relation between measured input and sensor output. When the input is $100 \%$ the expected output is $100 \%$. The actual output is the result of a linear effect where the output is proportional to the input but scaled. For example, if the input is $10 \mathrm{~g}$ and there is a $2 \%$ scale factor error, the output measurement will be $10.2 \mathrm{~g}$. It can also be expressed as a $20000 \mathrm{ppm}^{1}$ error [8]. As shown in plot (a) in Fig. 4.12, the value of error around $x$-axis is 0.9998 .
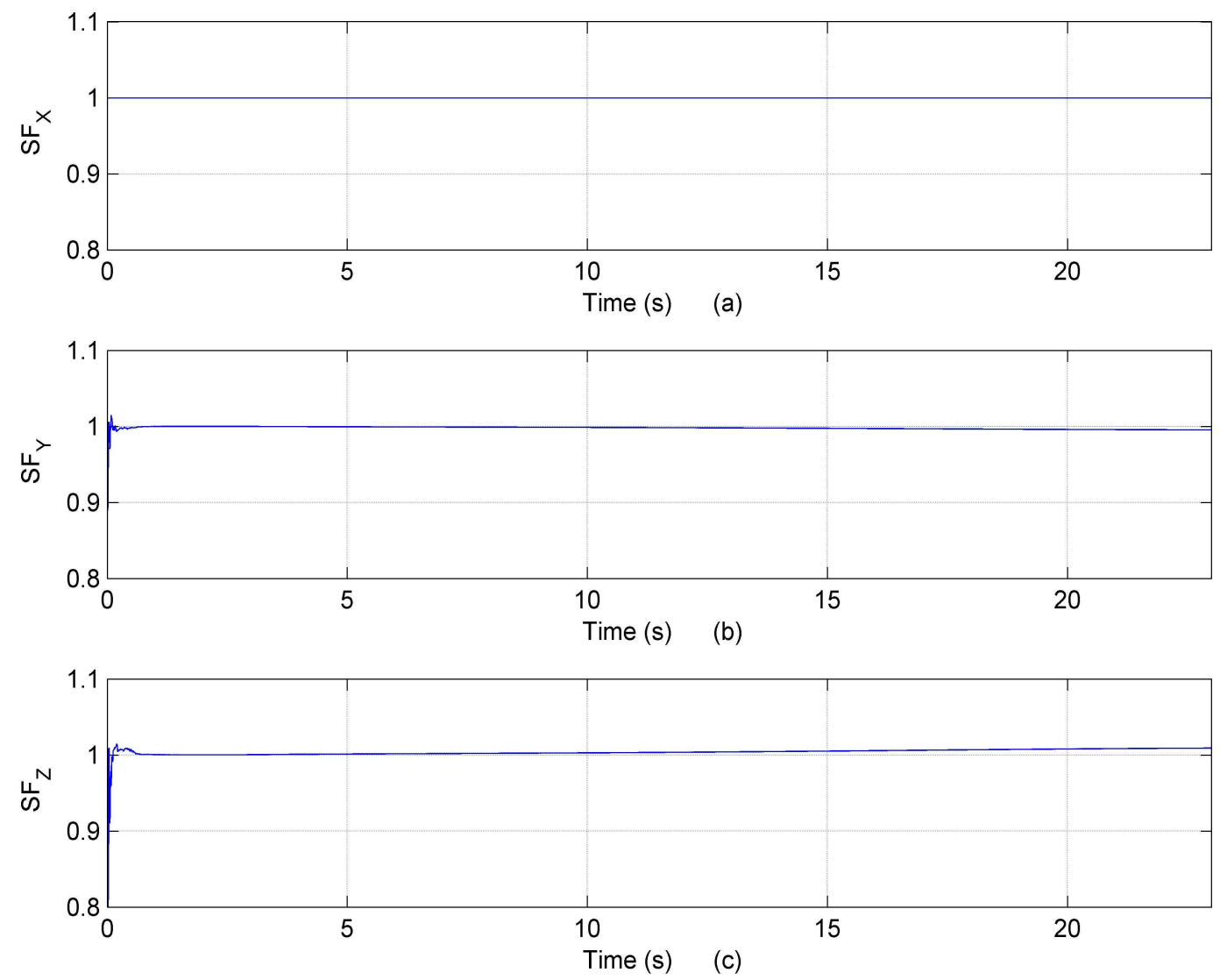

Figure 4.10: Scale Factor Estimation Along $x, y$, and $z$ Axes

\footnotetext{
${ }^{1} \mathrm{ppm}=$ part per million
} 


\subsubsection{Sensor Misalignment Estimations Along $\mathrm{x}, \mathrm{y}$, and $\mathrm{z}$ Axes}

Fig. 4.13 demonstrates the misalignment error along $x$-axis, $y$-axis, and $z$-axis. This misalignment error is generated due to the fact that the three accelerometers were not mounted orthogonally to each other. This leads to a correlation between sensors. For example, assume one axis is pointed perfectly up. The accelerometer on this axis is measuring gravity. If the other two axes were perfectly orthogonal, they do not measure any of the effect of gravity. If there is a nonorthogonality, the other axes also measure gravity, leading to a correlation in the measurements. For each axis, there are two misalignment errors [8]. The graph (a) in Fig. 4.13 shows that the misalignment error around $x$-axis with $y$-axis is 0.00243 Radian, and the graph (b) in Fig. 4.13 shows the misalignment error around $x$-axis with $z$-axis is 0.0124 Radian which they are less than the tolerance of the sensor errors. 

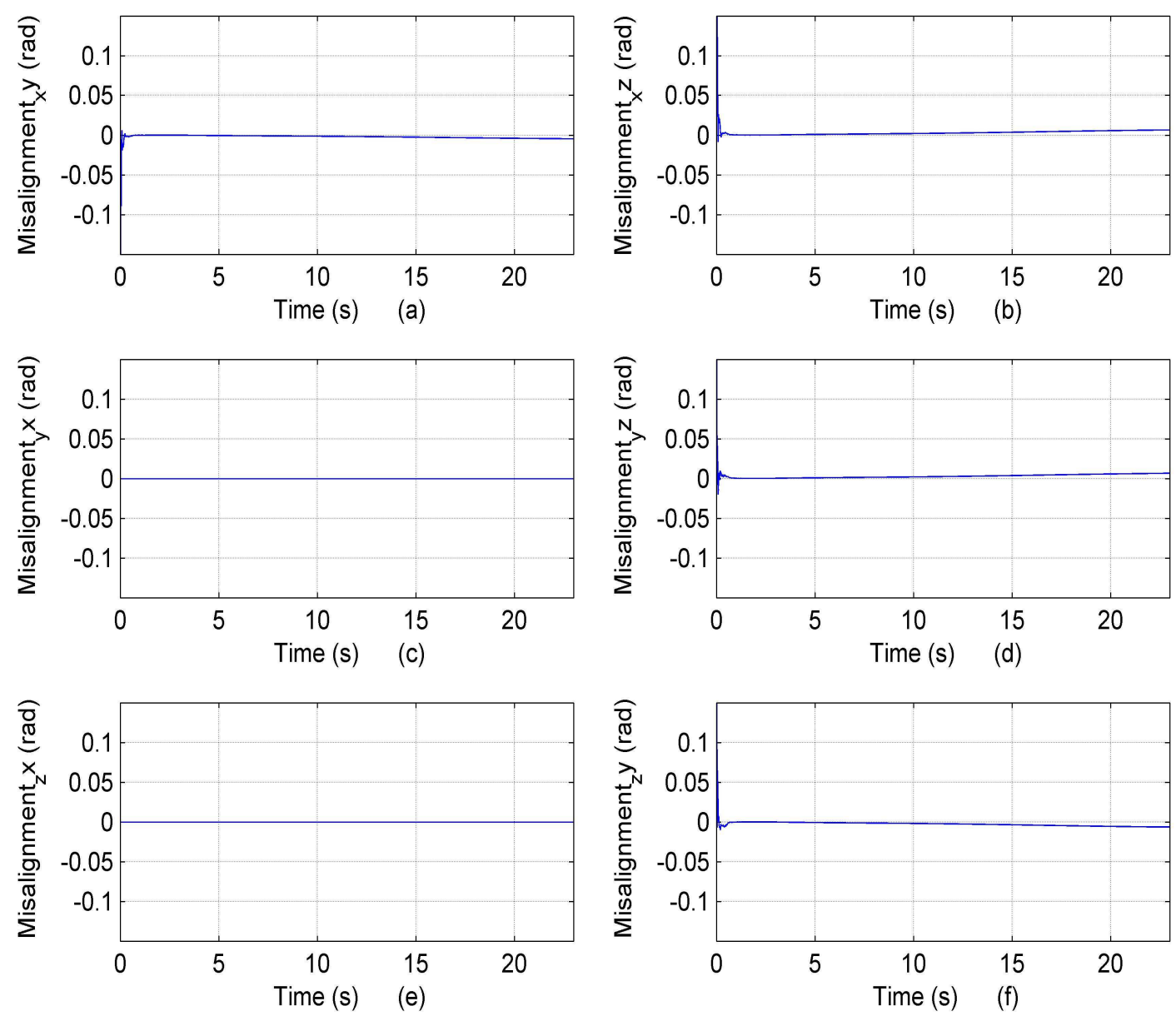

Figure 4.11: Sensor Misalignment Estimations Along $x, y$, and $z$ Axes 
Table 4.2: Summary of 3-D testing results

\begin{tabular}{|c|c|c|}
\hline Sensor axis & Bias (g) & $\begin{array}{c}\text { Sensor } \\
\text { Specs }\end{array}$ \\
\hline $\mathrm{x}$ & 0.0384 & \pm 0.05 \\
\hline $\mathrm{y}$ & 0.04902 & \pm 0.05 \\
\hline $\mathrm{z}$ & -0.05188 & \pm 0.05 \\
\hline Sensor axis & Scale factor & $\begin{array}{c}\text { Sensor } \\
\text { Specs }\end{array}$ \\
\hline $\mathrm{x}$ & 0.9998 & $1 \pm 0.01$ \\
\hline $\mathrm{y}$ & 1.003 & $1 \pm 0.01$ \\
\hline $\mathrm{z}$ & 1.018 & $1 \pm 0.01$ \\
\hline Axes & Misalignment & Sensor \\
couples & Radian) & \pm 0.0034 \\
\hline $\mathrm{xy}$ & 0.00243 & \pm 0.0034 \\
\hline $\mathrm{xz}$ & 0.0124 & \pm 0.0034 \\
\hline $\mathrm{yx}$ & -0.00170 & \pm 0.0034 \\
\hline $\mathrm{yz}$ & -0.01233 & \pm 0.0034 \\
\hline $\mathrm{zx}$ & -0.000262 & \pm 0.0034 \\
\hline $\mathrm{zy}$ & -0.00384 & \\
\hline
\end{tabular}

\subsection{Effect the Misalignment of the Turntable on the Results}

In section 3.5.2, it was verified that there was small misalignment in the turntable. The maximum misalignment of the turntable with the accelerometer position was 0.0015 Radian at point 4 (refer to Fig. 3.9). However, this value will not affect the result of the misalignment of the $3 \mathrm{D}$ accelerometer testing system because the $45^{\circ}$ mounting block was leveled with the vertical axis of the turntable to be zero. In other words, the mounting block was balanced independently to the turntable. 


\section{Chapter 5}

\section{Conclusion, Recommendation and Future Works.}

\section{$5.1 \quad$ Summary and Conclusion}

The 3D Axis turntable prototype has successfully conducted all the required tests for MEMS accelerometer sensor. Thus, it proves that high torque DC motor with a speed controller is able to provide a range of acceleration up to $\pm 50 \mathrm{~g}$. The high resolution encoder (524288 counts/rev) is sufficient to measure the whole range of angular velocity inputs. There is no any distortion in the signal when it goes through the slip rings. Hence, This testing methodology has determined all the sensor errors in the range of its specifications. For 3-D testing, the testing data were collected within 20-25 sec. The estimation of sensor errors parameter converged within 30 sec. Moreover, the new system should be able to test many accelerometer sensor at a time. This feature will decrease the costs of the total testing time. Furthermore, the new system should be able to test a sensor along multiple axes at once. This process will lead to increase the accuracy of the testing system because it does not need to reposition the accelerometer sensor during the test. Some errors parameters are beyond the speciation provide by the manufacture because of the alignment of the machine was not perfectly calibrated. Therefor, further investigation is needs. 


\subsection{Future work and recommendation}

MEMS accelerometer sensor is the device that is temperature sensitive. Therefore, it could be better to implement the temperature effect on the sensor to employ it for environmental temperature simulation. Every test has to be conducted through the whole sensor operation temperature range. 


\section{References}

[1] Siva Prasad. Design Simulation Fabrication of Micromachined Acceleration Sensor. PhD thesis, Jawaharlal Nehru Technological University, 2010.

[2] Grigori, Teodor Lucian, and Negrea. About the Capacitive Sensing of the Acceleration in Miniaturized Strap-down Inertial Navigation Systems. pages 210-217, 2013.

[3] Mark Lemkin and Bernhard E Boser. A Three Axis Micromachined Accelerometer with a CMOS Position Sense Interface and Digital Offset Trim Electronics. pages 456-468, 1999.

[4] Steven J Miller. The Method of Least Squares. pages 1-7, 2006.

[5] NA Thacker and AJ Lacey. Tutorial: The Likelihood Interpretation of the Kalman Filter. TINA Memos: Advanced Applied Statistics, 2(1):1-11, 1996.

[6] Toshiaki Tsuji, Takuya Hashimoto, Hiroshi Kobayashi, Mariko Mizuochi, and Kouhei Ohnishi. A Wide-range Velocity Measurement Method for Motion Control. IEEE Transactions on industrial electronics, 56(2):510-519, 2009.

[7] Howard Curtis. Orbital Mechanics for Engineering Students. ButterworthHeinemann, 2013.

[8] W Flenniken, J Wall, and D Bevly. Characterization of Various IMU Error Sources and the Effect on Navigation Performance. In ION GNSS, pages 967978, 2005.

[9] Matej Andrejašic. MEMS Accelerometers. PhD thesis, University of Ljubljana, 2008.

[10] Andrea Nisticò. Working Principle of a Capacitive Accelerometer. PhD thesis, University of Roma Tor Vergata, 2013.

[11] Jonathan Bernstein. Overview of MEMS Inertial Sensing Technology. Available at http://www.sensorsmag.com/sensors/acceleration-vibration/ an-overview-mems-inertial-sensing-technology-970. 
[12] Pradeep Chakraborty2 comments. MEMS Market Overview. Available at https: //pradeepchakraborty.wordpress.com/category/jeremie-bouchaud/.

[13] Sascha Revel. Overview of Testing Techniques and Consideration. Available at http://www.memsjournal.com/2011/09/ inertial-mems-devices-an-overview-of-testing-techniques-and.html.

[14] IEEE Aerospace and Electronic Systems Society. Recommendation Preactice for Precision Centrifuge Testing of Linear Accelerometers., 9 2001. Rev.836-2001.

[15] Douglas C Giancoli. General Physics. Prentice-Hall Englewood Cliffs, NJ, 1984.

[16] Mohinder S Grewal, Lawrence R Weill, and Angus P Andrews. Global Positioning Systems, Inertial Navigation, and Integration. John Wiley \& Sons, 2007.

[17] Huy Le. Development of Methodology and Equipment for Multi-axis MEMS Gyroscope Test. Master's thesis, Portland State University, Portland, 2011.

[18] Grantham Pang and Hugh Liu. Evaluation of a low-cost mems Accelerometer for Distance Measurement. Journal of Intelligent and Robotic Systems, 30(3):249$265,2001$.

[19] Tony Lacey. Tutorial: The Kalman Filter. Georgia Institute of Technology.

[20] Jan K Bekkeng. Calibration of a Novel MEMS Inertial Reference Unit. IEEE Transactions on instrumentation and measurement, 58(6):1967-1974, 2009.

[21] Analog Devices. Triaxial Inertial Sensor with Magnetometer adis16400. NCSL International Workshop Symposium, 2015.

[22] GPI. Virtual Absolute Technology. Available at http://gurley.com/Virtual_ Absolute/VAoverview.htm.

[23] Hejun Du and Christine Connolly. Technological Improvements in Position Sensing. Sensor Review, 27(1):17-23, 2007. 


\section{Appendix A}

\section{Glossary of MEMS Accelerometer Testing Machine Terminology}

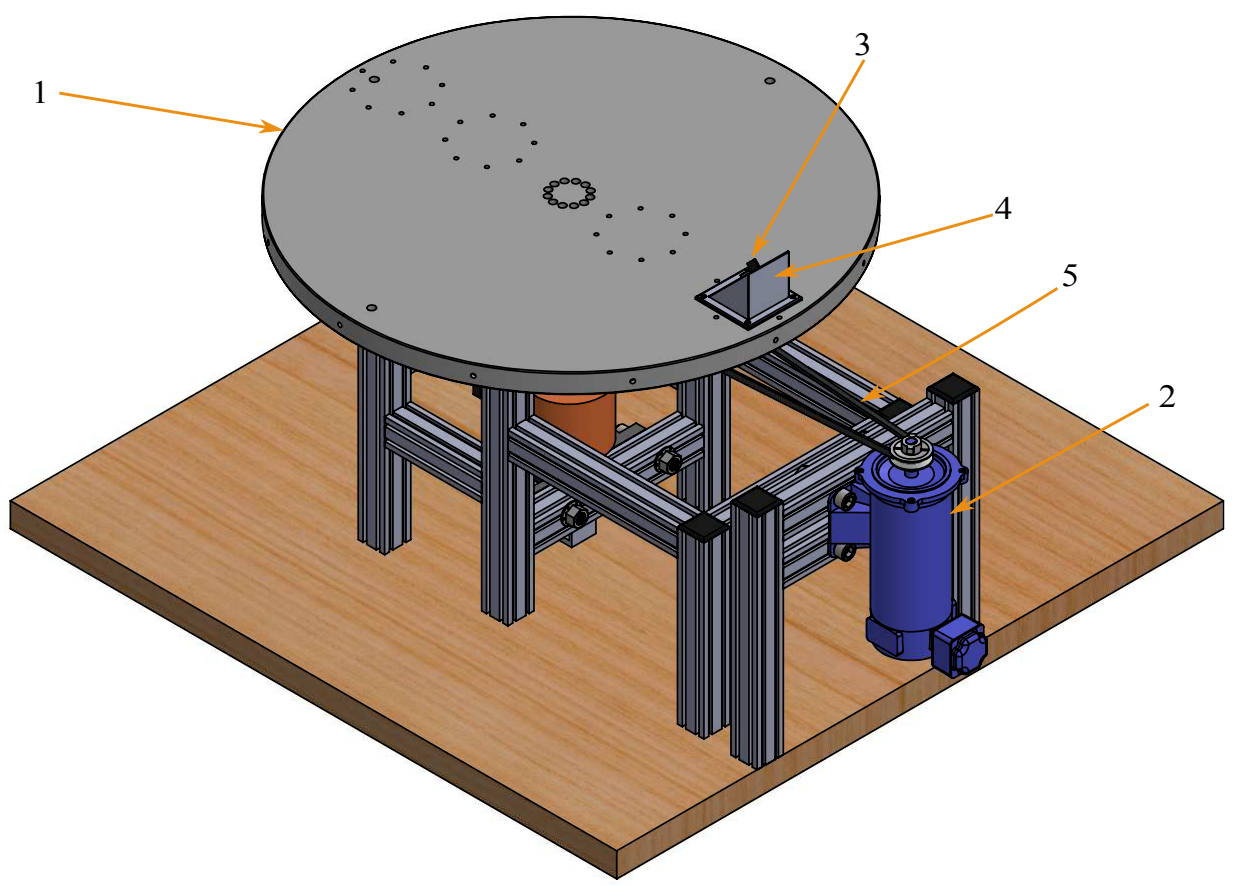

Figure A.1: Multi-axes MEMS Accelerometer Testing Machine

1: Turntable. 
2: DC Motor.

3: block $45^{\circ}$.

4: MEMS Sensor.

5: Belt.

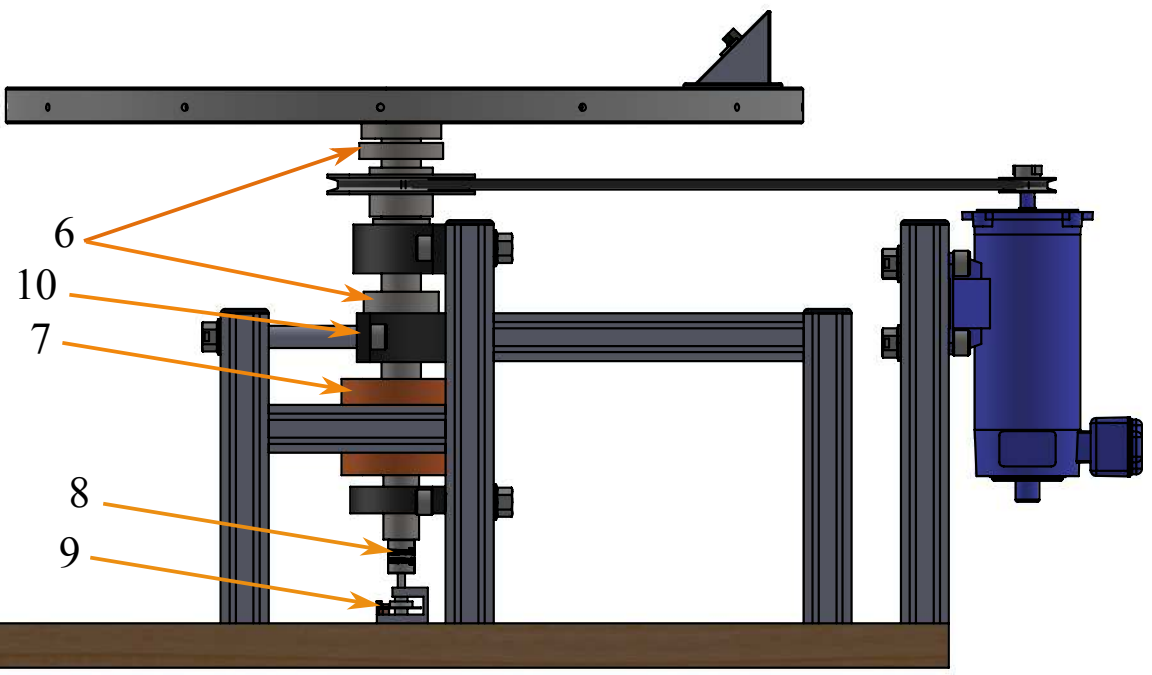

Figure A.2: Multi-axes MEMS Accelerometer Testing Machine

6: Shaft.

7: Slip Ring.

8: Shaft Coupling.

9: Encoder.

10: Bearings 


\section{Appendix B}

\section{Labview ${ }^{M T}$ Program}

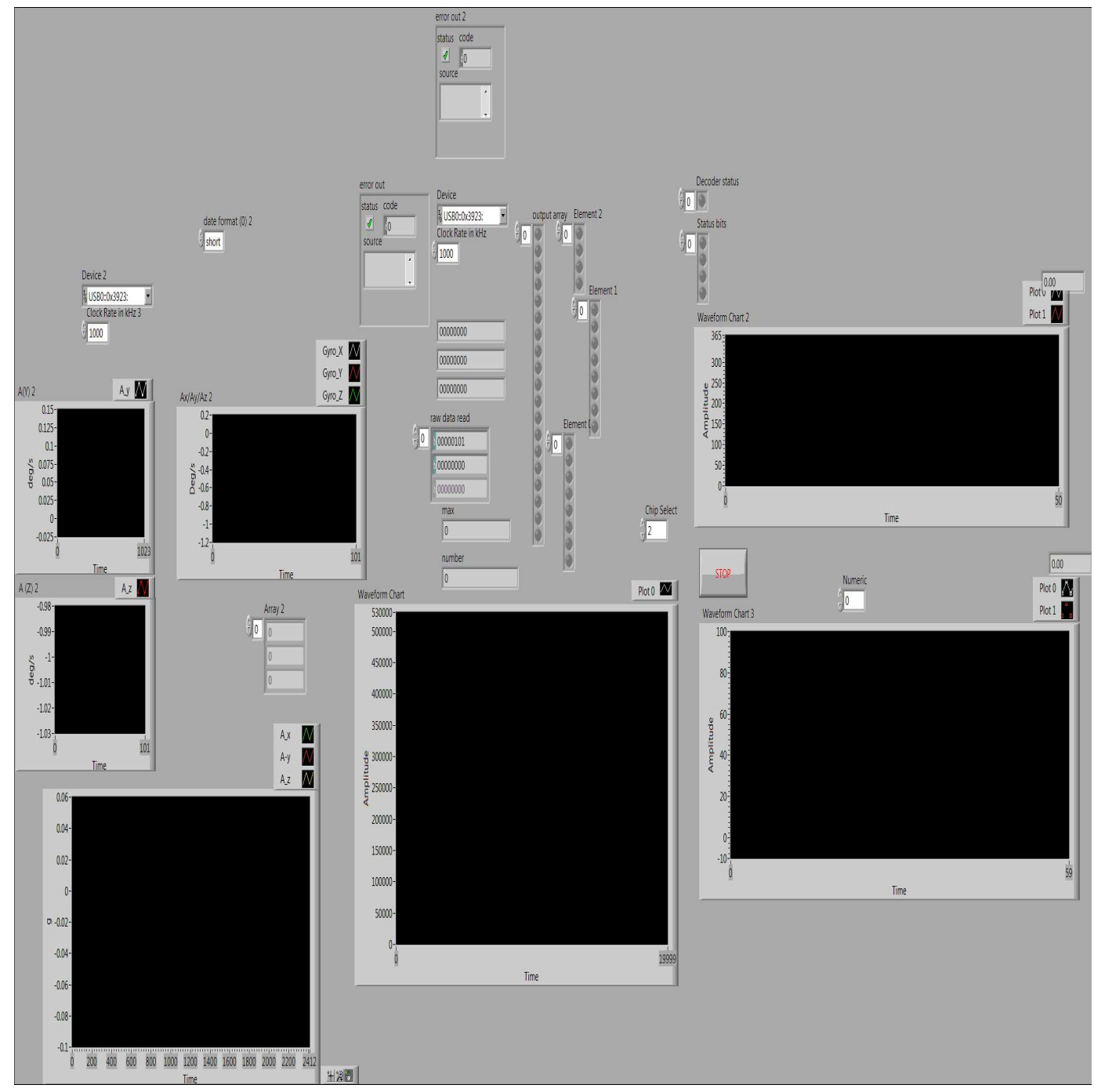

Figure B.1: Labview ${ }^{M T}$ Program 


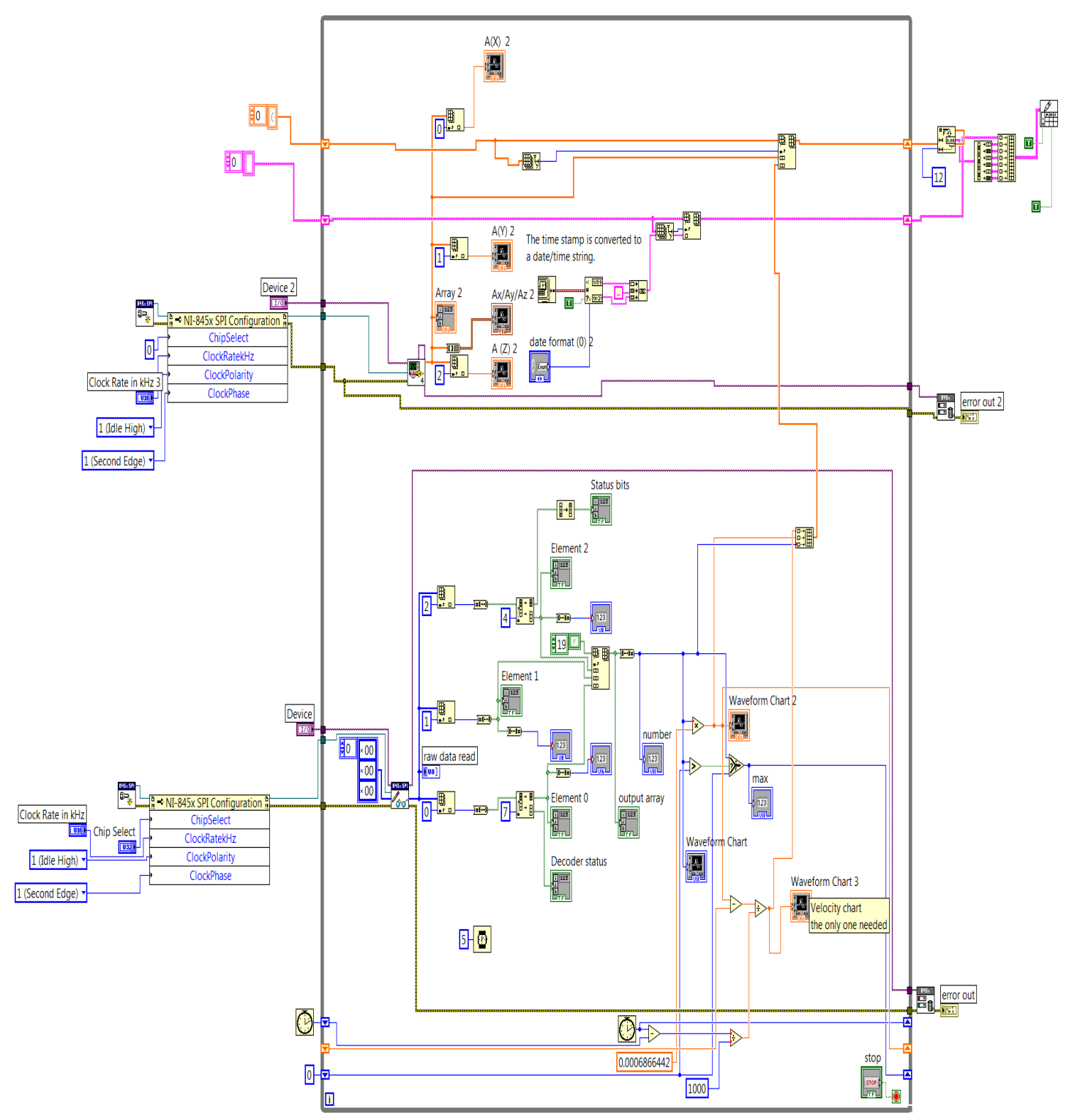

Figure B.2: The Block Diagram for 3D Accelerometer Testing 


\section{Appendix C}

\section{Kalman Filter Parameters of the Sensor}

$$
\begin{aligned}
& \mathbf{A}=\left[\begin{array}{lll}
1 & \mathrm{dt} ; & 0
\end{array}\right] \\
& \mathrm{dt}=0.016 \\
& \text { Noise density }=0.05 \\
& \mathrm{BW}=330 \\
& \text { sigma }=\text { Noise density*sqrt }(\mathrm{BW} * 1.6) \\
& \mathbf{R}=\operatorname{sigma}{ }^{2} \\
& \mathbf{Q}=0.00001^{*} \text { eye }(2) \\
& \mathbf{H}=\left[\begin{array}{ll}
1 & 0
\end{array}\right]
\end{aligned}
$$

